Pacific

Journal of

Mathematics

CONVEX PBW-TYPE LYNDON BASIS AND RESTRICTED TWO-PARAMETER QUANTUM GROUPS OF TYPE $\boldsymbol{G}_{2}$

NAIHONG Hu AND XIULING WANG 


\title{
CONVEX PBW-TYPE LYNDON BASIS AND RESTRICTED TWO-PARAMETER QUANTUM GROUPS OF TYPE $G_{2}$
}

\author{
NAIHONG Hu AND XiUling WANG
}

\begin{abstract}
From the 2-parameter quantum group $U_{r, s}\left(G_{2}\right)$ defined by $\mathrm{Hu}$ and Shi in 2007, we construct finite-dimensional pointed Hopf algebras $\mathfrak{u}_{r, s}\left(G_{2}\right)$ (that is, restricted 2-parameter quantum groups); these turn out to be Drinfel'd doubles. Crucial is a detailed combinatorial construction of the convex PBW-type Lyndon basis for type $G_{2}$ in the 2-parameter quantum version. We exhibit the possible commutation relations among quantum root vectors. Then we show that the restricted quantum groups are ribbon Hopf algebras under certain conditions, by determining their left and right integrals. We also determine all the Hopf algebra isomorphisms of $\mathfrak{u}_{r, s}\left(G_{2}\right)$ by describing its sets of left (right) skew-primitive elements.
\end{abstract}

\section{Introduction}

In 1941, H. Hopf first observed in algebraic topology what are now known as Hopf algebras. Since then, numerous mathematicians have studied these algebras as purely algebraic objects, and applied them to other areas of mathematics, such as Lie theory, knot theory and combinatorics. A long standing problem in the area is the full classification of the finite-dimensional Hopf algebras. One of the very few general classification results says that any cocommutative Hopf algebra over the complex field $\mathbb{C}$ is a semidirect product of the universal enveloping algebra of a Lie algebra and a group algebra; this is the Cartier-Kostant-Milnor-Moore theorem. Since Kaplansky's ten conjectures [1975] have stimulated much research on Hopf algebras, and there have been many significant advances during the last two decades. A rich set of examples of noncommutative and noncocommutative Hopf algebras is supplied by the Drinfel'd-Jimbo quantum groups $U_{q}(\mathfrak{g})$, where $\mathfrak{g}$

MSC2000: primary 17B37, 81R50; secondary 17B35.

Keywords: restricted 2-parameter quantum groups, Lyndon basis, Drinfel'd double, integrals, ribbon Hopf algebra.

$\mathrm{Hu}$ is supported in part by the NNSF (grants 10431040 and 10728102), the PCSIRT and the PDPF from the MOE of China, the National/Shanghai Priority Academic Discipline Programmes (project number B407). Wang is supported by the Nankai Research-encouraging Fund for PhD Teachers and a fund from the LPMC.

Wang is the corresponding author. 
is a semisimple Lie algebra (see [Drinfel'd 1987]), which were found in the mid 1980 s, and by the finite-dimensional small quantum groups $\mathfrak{u}_{q}(\mathfrak{g})$, where $q=\epsilon$ is a root of unity; the latter were introduced by Lusztig [1990a].

Until now, the classification of Hopf algebras has split into the semisimple case and the nonsemisimple case. Montgomery [1998] provides a good overview of the first. The classification in the second focuses on pointed Hopf algebras over an algebraically closed field of characteristic 0; see [Andruskiewitsch and Schneider 2006; 2002]. Pointed Hopf algebras play an important role in [Andruskiewitsch and Schneider 1998; Beattie et al. 1999; Gelaki 1998], where Kaplansky's 10th conjecture is refuted by constructing infinitely many nonisomorphic Hopf algebras of a given prime power dimension.

Since finite-dimensional Hopf algebras are far from being classified, it is meaningful to have various means of constructing examples of them (see for example [Andruskiewitsch and Schneider 1998; 2002; Benkart and Witherspoon 2004b; Hu 2000; 2004; Hu and Wang 2007; 2009; Lusztig 1990a; Radford 1976; Taft 1971]. Interestingly to us, Benkart and Witherspoon [2004b] and Hu and Wang [2009] determined the structure of restricted 2-parameter quantum groups $\mathfrak{u}_{r, s}\left(\mathfrak{s l}_{n}\right)$ and $\mathfrak{u}_{r, s}\left(\mathfrak{s o}_{2 n+1}\right)$, respectively, when both parameters $r$ and $s$ are roots of unity; both are new finite-dimensional pointed Hopf algebras and have new ribbon elements under some conditions. These results will act as a starting point for further studying the representation theory of 2-parameter quantum groups at roots of unity, as in the 1-parameter setting; see [De Concini and Kac 1990; Lusztig 1990a]. Our goal here is to solve the same problems for the type $G_{2}$ case.

Since the work of Drinfel'd [1987] and Jimbo [1986], work on 2- or multiparameter quantum groups has focused on quantized function algebras and quantum enveloping algebras. Work in the 1990s focused mainly on the type $A$ cases. In 2001, motivated by the down-up algebras approach of [Benkart 1999], Benkart and Witherspoon [2004c] reobtained Takeuchi's definition of the 2-parameter quantum groups of $\mathfrak{g l}_{n}$ and $\mathfrak{s l}_{n}$. Since then, a systematic study of the 2-parameter quantum groups of type $A$ has been developed by Benkart and Witherspoon and their collaborators Kang and Lee; see [Benkart et al. 2003; 2006; Benkart and Witherspoon 2004a; 2004b]. In 2004, Bergeron, Gao and Hu [2006] defined the 2-parameter quantum groups $U_{r, s}(\mathfrak{g})$ (in the sense of Benkart and Witherspoon) for $\mathfrak{g}=\mathfrak{s o}_{2 n+1}, \mathfrak{s o}_{2 n}$ and $\mathfrak{s p}_{2 n}$, which are realized as Drinfel'd doubles, and described weight modules in the category 0 when $r s^{-1}$ is not a root of unity; see [Bergeron et al. 2007]. Afterwards, $\mathrm{Hu}$ and his collaborators continued to develop the corresponding theory for exceptional types $G$ and $E$ and the affine cases; see [Hu and Shi 2007; Bai and Hu 2008; Hu et al. 2008; Hu and Zhang 2006].

It should be mentioned that, unlike in the 1-parameter case (see [Kassel 1995; Lusztig 1990b; 1993]), one cannot write out conveniently the convex PBW-type 
bases for 2-parameter quantum groups in terms of Lusztig's braid group actions. This is seen in [Bergeron et al. 2006, Theorem 3.1] in the study of Lusztig's symmetry, and is one of difficulties encountered in the 2-parameter setting. The combinatorial construction of the PBW-type bases in the quantum setup is rather nontrivial, as it depends not only on the choice of a convex ordering on a positive root system [Beck 1994; Rosso 2002], but also on the corresponding inserting manner of q-bracketings (see [Rosso 2002; Kharchenko 1999; 2002; Bai and $\mathrm{Hu}$ 2008; Hu et al. 2008]) into good Lyndon words; see also [Lalonde and Ram 1995]. The construction of convex PBW-type bases in the 2-parameter quantum cases has been given for type $A$ in [Benkart et al. 2003], type $E$ in [Bai and Hu 2008], for low rank cases of types $B, C, D$ in [Hu 2005], and for type $B$ cases of arbitrary rank in [Hu and Wang 2009]. Motivated by [Hu 2005; Hu and Wang 2009; Lalonde and Ram 1995; Rosso 2002], we will first explicitly construct the convex PBW-type Lyndon bases of type $G_{2}$; this will be a prerequisite for later discussions.

The main purpose of this paper is to construct a family of $\ell^{16}$-dimensional pointed Hopf algebras $\mathfrak{u}_{r, s}\left(G_{2}\right)$ as a quotient of $U_{r, s}\left(G_{2}\right)$ by a Hopf ideal $\Phi$; these algebras are generated by certain homogeneous central elements of degree $\ell$, where $r$ is a primitive $d$-th root of unity, $s$ is a primitive $d^{\prime}$-th root of unity, and $\ell$ is the least common multiple of $d$ and $d^{\prime}$. We will assume that the ground field $\mathbb{K}$ contains a primitive $\ell$-th root of unity, and then show that the restricted quantum group $\mathfrak{u}_{r, s}\left(G_{2}\right)$ is a ribbon Hopf algebra. Our treatments in type $G_{2}$ are complicated by the complexity of Lyndon bases corresponding to nonsimply-laced Dynkin diagrams.

The article is organized as follows. In Section 2, we recall the definition of the 2-parameter quantum groups of type $G_{2}$, and some basics about their structure. In particular, we directly construct the convex PBW-type Lyndon bases for $U_{r, s}\left(G_{2}\right)$. In Section 3, we first exhibit the possible commutation relations, and then determine those central elements of degree $\ell$ that generate a Hopf ideal in the case when both parameters $r$ and $s$ are roots of unity. These enable us to further derive the restricted 2-parameter quantum groups $\mathfrak{u}_{r, s}\left(G_{2}\right)$. In Section 4, we show that the Hopf algebra $\mathfrak{u}_{r, s}\left(G_{2}\right)$ is pointed, and determine all its Hopf algebra isomorphisms by describing its set of left (right) skew-primitive elements. In the type $A$ case, Benkart and Witherspoon missed some important left (right) skewprimitive elements; see [2004b, (3.6) and (3.7)]. Restoring these elements leads to new, interesting families of isomorphisms. In Section 5, we show that $\mathfrak{u}_{r, s}\left(G_{2}\right)$ is a Drinfel'd double of a certain (Borel-type) Hopf subalgebra $\mathfrak{b}$. In Section 6, we determine the left and right integrals of $\mathfrak{b}$ and use them in combination with a result of Kauffmann and Radford [1993] to characterize $\mathfrak{u}_{r, s}\left(G_{2}\right)$ as a ribbon Hopf algebra. 


\section{2. $U_{r, s}\left(G_{2}\right)$ and the convex PBW-type Lyndon basis}

We recall the definition of 2-parameter quantum groups of type $G_{2}$, which were introduced by $\mathrm{Hu}$ and Shi [2007].

The 2-parameter quantum group $\boldsymbol{U}_{r, s}\left(\boldsymbol{G}_{2}\right)$. Let $\mathbb{K}=\mathbb{Q}(r, s)$ be a field of rational functions with two indeterminates $r$ and $s$, with $r^{3} \neq s^{3}$ and $r^{4} \neq s^{4}$. Let $\Phi$ be a finite root system of $G_{2}$, with $\Pi$ a base of simple roots that is a subset of Euclidean space $E=\mathbb{R}^{3}$ with an inner product $(\cdot, \cdot)$. Let $\epsilon_{1}, \epsilon_{2}, \epsilon_{3}$ denote an orthonormal basis of $E$. Then

$$
\begin{aligned}
& \Pi=\left\{\alpha_{1}=\epsilon_{1}-\epsilon_{2}, \alpha_{2}=\epsilon_{2}+\epsilon_{3}-2 \epsilon_{1}\right\}, \\
& \Phi= \pm\left\{\alpha_{1}, \alpha_{2}, \alpha_{2}+\alpha_{1}, \alpha_{2}+2 \alpha_{1}, \alpha_{2}+3 \alpha_{1}, 2 \alpha_{2}+3 \alpha_{1}\right\} .
\end{aligned}
$$

In this case, we set

$$
\begin{array}{ll}
r_{1}=r^{\left(\alpha_{1}, \alpha_{1}\right) / 2}=r, & s_{1}=s^{\left(\alpha_{1}, \alpha_{1}\right) / 2}=s, \\
r_{2}=r^{\left(\alpha_{2}, \alpha_{2}\right) / 2}=r^{3}, & s_{2}=s^{\left(\alpha_{2}, \alpha_{2}\right) / 2}=s^{3} .
\end{array}
$$

Definition 2.1. Let $U=U_{r, s}\left(G_{2}\right)$ be the associative algebra over $\mathbb{Q}(r, s)$ generated by symbols $e_{i}, f_{i}, \omega_{i}^{ \pm 1}, \omega_{i}^{\prime \pm 1}$ for $i=1,2$ subject to the relations (G1)-(G6):

$$
\begin{aligned}
{\left[\omega_{i}^{ \pm 1}, \omega_{j}^{ \pm 1}\right]=\left[\omega_{i}^{ \pm 1}, \omega_{j}^{\prime \pm 1}\right]=\left[\omega_{i}^{ \pm 1}, \omega_{j}^{ \pm 1}\right]=0, } & \omega_{i} \omega_{i}^{-1}=1=\omega_{j}^{\prime} \omega_{j}^{-1} . \\
\omega_{1} e_{1} \omega_{1}^{-1}=\left(r s^{-1}\right) e_{1}, & \omega_{1} f_{1} \omega_{1}^{-1}=\left(r^{-1} s\right) f_{1}, \\
\omega_{1} e_{2} \omega_{1}^{-1}=s^{3} e_{2}, & \omega_{1} f_{2} \omega_{1}^{-1}=s^{-3} f_{2}, \\
\omega_{2} e_{1} \omega_{2}^{-1}=r^{-3} e_{1}, & \omega_{2} f_{1} \omega_{2}^{-1}=r^{3} f_{1}, \\
\omega_{2} e_{2} \omega_{2}^{-1}=\left(r^{3} s^{-3}\right) e_{2}, & \omega_{2} f_{2} \omega_{2}^{-1}=\left(r^{-3} s^{3}\right) f_{2} . \\
\omega_{1}^{\prime} e_{1} \omega_{1}^{\prime-1}=\left(r^{-1} s\right) e_{1}, & \omega_{1}^{\prime} f_{1} \omega_{1}^{\prime-1}=\left(r s^{-1}\right) f_{1}, \\
\omega_{1}^{\prime} e_{2} \omega_{1}^{\prime-1}=r^{3} e_{2}, & \omega_{1}^{\prime} f_{2} \omega_{1}^{-1}=r^{-3} f_{2}, \\
\omega_{2}^{\prime} e_{1} \omega_{2}^{\prime-1}=s^{-3} e_{1}, & \omega_{2}^{\prime} f_{1} \omega_{2}^{-1}=s^{3} f_{1}, \\
\omega_{2}^{\prime} e_{2} \omega_{2}^{\prime-1}=\left(r^{-3} s^{3}\right) e_{2}, & \omega_{2}^{\prime} f_{2} \omega_{2}^{\prime-1}=\left(r^{3} s^{-3}\right) f_{2} .
\end{aligned}
$$

(G4) For $1 \leq i, j \leq 2$, we have $\left[e_{i}, f_{j}\right]=\delta_{i j}\left(\omega_{i}-\omega_{i}^{\prime}\right) /\left(r_{i}-s_{i}\right)$.

(G5) We have the $(r, s)$-Serre relations

(G5) ${ }_{1} \quad e_{2}^{2} e_{1}-\left(r^{-3}+s^{-3}\right) e_{2} e_{1} e_{2}+(r s)^{-3} e_{1} e_{2}^{2}=0$,

$(\mathrm{G} 5)_{2} e_{1}^{4} e_{2}-(r+s)\left(r^{2}+s^{2}\right) e_{1}^{3} e_{2} e_{1}+r s\left(r^{2}+s^{2}\right)\left(r^{2}+r s+s^{2}\right) e_{1}^{2} e_{2} e_{1}^{2}$

$$
-(r s)^{3}(r+s)\left(r^{2}+s^{2}\right) e_{1} e_{2} e_{1}^{3}+(r s)^{6} e_{2} e_{1}^{4}=0 .
$$


(G6) We have the $(r, s)$-Serre relations

$$
\begin{aligned}
& f_{1} f_{2}^{2}-\left(r^{-3}+s^{-3}\right) f_{2} f_{1} f_{2}+(r s)^{-3} f_{2}^{2} f_{1}=0 \\
& f_{2} f_{1}^{4}-(r+s)\left(r^{2}+s^{2}\right) f_{1} f_{2} f_{1}^{3}+r s\left(r^{2}+s^{2}\right)\left(r^{2}+r s+s^{2}\right) f_{1}^{2} f_{2} f_{1}^{2} \\
& -(r s)^{3}(r+s)\left(r^{2}+s^{2}\right) f_{1}^{3} f_{2} f_{1}+(r s)^{6} f_{1}^{4} f_{2}=0 .
\end{aligned}
$$

The algebra $U_{r, s}\left(G_{2}\right)$ is a Hopf algebra, where the $\omega_{i}^{ \pm 1}$ and $\omega_{i}^{\prime \pm 1}$ are group-like elements, and the remaining Hopf structure is given by

$$
\begin{aligned}
\Delta\left(e_{i}\right) & =e_{i} \otimes 1+\omega_{i} \otimes e_{i}, & \Delta\left(f_{i}\right) & =1 \otimes f_{i}+f_{i} \otimes \omega_{i}^{\prime}, \\
\varepsilon\left(\omega_{i}^{ \pm 1}\right) & =\varepsilon\left(\omega_{i}^{\prime \pm 1}\right)=1, & \varepsilon\left(e_{i}\right) & =\varepsilon\left(f_{i}\right)=0, \\
S\left(\omega_{i}^{ \pm 1}\right) & =\omega_{i}^{\mp 1}, & S\left(\omega_{i}^{\prime \pm 1}\right) & =\omega_{i}^{\prime \mp 1}, \\
S\left(e_{i}\right) & =-\omega_{i}^{-1} e_{i}, & S\left(f_{i}\right) & =-f_{i}{\omega_{i}^{\prime}}^{-1} .
\end{aligned}
$$

When $r=q=s^{-1}$, the Hopf algebra $U_{r, s}\left(G_{2}\right)$ modulo the Hopf ideal generated by $\omega_{i}^{\prime}-\omega_{i}^{-1}$ for $i=1,2$ is just the Drinfel'd-Jimbo-type quantum group $U_{q}\left(G_{2}\right)$.

In any Hopf algebra $\mathscr{H}$, there exist the left- and the right-adjoint action defined by its Hopf algebra structure as

$$
\operatorname{ad}_{l} a(b)=\sum_{(a)} a_{(1)} b S\left(a_{(2)}\right) \quad \text { and } \quad \operatorname{ad}_{r} a(b)=\sum_{(a)} S\left(a_{(1)}\right) b a_{(2)},
$$

where $\Delta(a)=\sum_{(a)} a_{(1)} \otimes a_{(2)} \in \mathcal{H} \otimes \mathscr{H}$ for any $a, b \in \mathscr{H}$.

From the viewpoint of adjoint actions, the $(r, s)$-Serre relations (G5) and (G6) take the simplest forms

$\left(\operatorname{ad}_{l} e_{i}\right)^{1-a_{i j}}\left(e_{j}\right)=0$ for any $i \neq j \quad$ and $\quad\left(\operatorname{ad}_{r} f_{i}\right)^{1-a_{i j}}\left(f_{j}\right)=0$ for any $i \neq j$.

Let $U^{+}$and $U^{-}$be the subalgebras of $U=U_{r, s}\left(G_{2}\right)$ generated by the elements $e_{i}$ and $f_{i}$, respectively, for $i=1,2$. Let $\mathscr{B}$ and $\mathscr{B}^{\prime}$ denote the Hopf subalgebras of $U_{r, s}\left(G_{2}\right)$ generated by $e_{j}, \omega_{j}^{ \pm 1}$ and $f_{j}, \omega_{j}^{ \pm 1}$, respectively, for $j=1,2$.

Proposition 2.2. There exists a unique skew-dual pairing $\langle\cdot, \cdot\rangle: \mathscr{B}^{\prime} \times \mathscr{B} \rightarrow \mathbb{Q}(r, s)$ of the Hopf subalgebras $\mathscr{B}$ and $\mathscr{B}^{\prime}$ such that

$$
\begin{array}{rlrl}
\left\langle f_{i}, e_{j}\right\rangle & =\delta_{i j} /\left(s_{i}-r_{i}\right) & & \text { for } 1 \leq i, j \leq 2, \\
\left\langle\omega_{1}^{\prime}, \omega_{1}\right\rangle & =r s^{-1}, & \left\langle\omega_{1}^{\prime}, \omega_{2}\right\rangle=r^{-3}, \\
\left\langle\omega_{2}^{\prime}, \omega_{1}\right\rangle & =s^{3}, & \left\langle\omega_{2}^{\prime}, \omega_{2}\right\rangle=r^{3} s^{-3}, \\
\left\langle\omega_{i}^{\prime \pm 1}, \omega_{j}^{-1}\right\rangle & =\left\langle\omega_{i}^{ \pm 1}, \omega_{j}\right\rangle^{-1}=\left\langle\omega_{i}^{\prime}, \omega_{j}\right\rangle^{\mp 1} \quad \text { for } 1 \leq i, j \leq 2,
\end{array}
$$

and all other pairs of generators are 0 . Also, we have $\langle S(a), S(b)\rangle=\langle a, b\rangle$ for $a \in \mathscr{B}^{\prime}$ and $b \in \mathscr{B}$. 
Convex PBW-type Lyndon basis. Recall that a reduced expression of the longest element of Weyl group $W$ for type $G_{2}$ given by $w_{0}=s_{1} s_{2} s_{1} s_{2} s_{1} s_{2}$ yields a convex ordering of positive roots

$$
\alpha_{1}, \quad 3 \alpha_{1}+\alpha_{2}, \quad 2 \alpha_{1}+\alpha_{2}, \quad 3 \alpha_{1}+2 \alpha_{2}, \quad \alpha_{1}+\alpha_{2}, \quad \alpha_{2} .
$$

A positive root system is $\Phi^{+}=\left\{\alpha_{1}, 3 \alpha_{1}+\alpha_{2}, 2 \alpha_{1}+\alpha_{2}, 3 \alpha_{1}+2 \alpha_{2}, \alpha_{1}+\alpha_{2}, \alpha_{2}\right\}$.

Note that the above ordering also corresponds to the standard Lyndon tree of type $G_{2}$ :

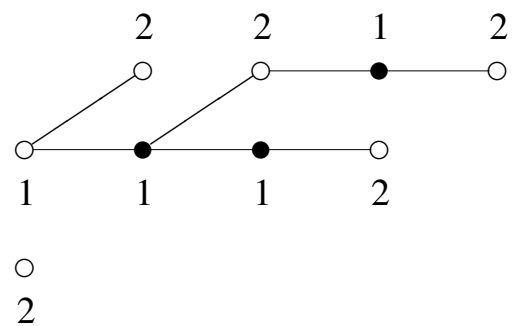

With the ordering and notation above, we can inductively define the quantum root vectors $E_{\alpha}$ in $U^{+}$as follows. Briefly, we set $E_{i}=E_{\alpha_{i}}, E_{12}=E_{\alpha_{1}+\alpha_{2}}$, $E_{112}=E_{2 \alpha_{1}+\alpha_{2}}, E_{1112}=E_{3 \alpha_{1}+\alpha_{2}}$ and $E_{11212}=E_{3 \alpha_{1}+2 \alpha_{2}}$.

We define inductively

$$
\begin{aligned}
E_{1} & =e_{1}, \quad E_{2}=e_{2}, \\
E_{12} & =e_{1} e_{2}-s^{3} e_{2} e_{1}, \\
E_{112} & =e_{1} E_{12}-r s^{2} E_{12} e_{1}, \\
E_{1112} & =e_{1} E_{112}-r^{2} s E_{112} e_{1}, \\
E_{11212} & =E_{112} E_{12}-r^{2} s E_{12} E_{112} .
\end{aligned}
$$

Then the defining relations for $U^{+}$in (G5) can be reformulated as saying

$$
\begin{aligned}
E_{12} e_{2} & =r^{3} e_{2} E_{12}, \\
e_{1} E_{1112} & =r^{3} E_{1112} e_{1} .
\end{aligned}
$$

Remark 2.3. (i) The defining relations in (2-1)-(2-4) can be reformulated by the left-adjoint action defined by its Hopf algebra structure. For example, $E_{12}=\operatorname{ad}_{l} e_{1}\left(e_{2}\right)=e_{1} e_{2}-s^{3} e_{2} e_{1}$.

(ii) Note that

$$
\left(\left\langle\omega_{i}^{\prime}, \omega_{j}\right\rangle\right)_{2 \times 2}=\left(\begin{array}{cc}
r s^{-1} & r^{-3} \\
s^{3} & r^{3} s^{-3}
\end{array}\right)
$$


and set $p_{j i}=\left\langle\omega_{i}^{\prime}, \omega_{j}\right\rangle$. To get the quantum root vector $E_{\alpha}$ in $U^{+}$, we have to add $(r, s)$-brackets on each good Lyndon word obeying the defining rule as follows: $E_{\gamma}:=\left[E_{\alpha}, E_{\beta}\right]_{\left\langle\omega_{\beta}^{\prime}, \omega_{\alpha}\right\rangle}=E_{\alpha} E_{\beta}-\left\langle\omega_{\beta}^{\prime}, \omega_{\alpha}\right\rangle E_{\beta} E_{\alpha}$ for $\alpha, \gamma, \beta \in \Phi^{+}$, with $\alpha<\gamma<\beta$ in the convex ordering and $\gamma=\alpha+\beta$.

Kharchenko [1999] found a PBW-type basis for a Hopf algebra generated by an abelian group and a finite set of skew primitive elements such that the adjoint action of the group on the skew primitive generators is given by multiplication with a character. Once we construct the quantum root vectors as above, according to [Kharchenko 1999; Rosso 2002], we will have this result:

Theorem 2.4. $\left\{E_{2}^{n_{1}} E_{12}^{n_{2}} E_{11212}^{n_{3}} E_{112}^{n_{4}} E_{1112}^{n_{5}} E_{1}^{n_{6}} \mid n_{i} \in \mathbb{N}\right\}$ forms a Lyndon basis of the algebra $U^{+}$.

Recall that $\tau$ (see [Hu et al. 2008]) is a $\mathbb{Q}$-antiautomorphism of $U_{r, s}\left(G_{2}\right)$ such that $\tau(r)=s, \tau(s)=r, \tau\left(\left\langle\omega_{i}^{\prime}, \omega_{j}\right\rangle^{ \pm 1}\right)=\left\langle\omega_{j}^{\prime}, \omega_{i}\right\rangle^{\mp 1}$, and

$$
\tau\left(e_{i}\right)=f_{i}, \quad \tau\left(f_{i}\right)=e_{i}, \quad \tau\left(\omega_{i}\right)=\omega_{i}^{\prime}, \quad \tau\left(\omega_{i}^{\prime}\right)=\omega_{i} .
$$

Using $\tau$ to $U^{+}$, we can get the negative quantum root vectors in $U^{-}$. Define $F_{i}=\tau\left(E_{i}\right)=f_{i}$ for $1 \leq i \leq 2$, and

$$
\begin{aligned}
F_{12} & =\tau\left(E_{12}\right)=f_{2} f_{1}-r^{3} f_{1} f_{2}, \\
F_{112} & =\tau\left(E_{112}\right)=F_{12} f_{1}-r^{2} s f_{1} F_{12}, \\
F_{1112} & =\tau\left(E_{1112}\right)=F_{112} f_{1}-r s^{2} f_{1} F_{112}, \\
F_{11212} & =\tau\left(E_{11212}\right)=F_{12} F_{112}-r s^{2} F_{112} F_{12} .
\end{aligned}
$$

Corollary 2.5. $\left\{F_{1}^{m_{1}} F_{1112}^{m_{2}} F_{112}^{m_{3}} F_{11212}^{m_{4}} F_{12}^{m_{5}} F_{2}^{m_{6}} \mid m_{i} \in \mathbb{N}\right\}$ forms a Lyndon basis of the algebra $U^{-}$.

\section{Restricted 2-parameter quantum groups}

From now on, we restrict the parameters $r$ and $s$ to be roots of unity: $r$ is a primitive $d$-th root of unity, $s$ is a primitive $d^{\prime}$-th root of unity, and $\ell$ is the least common multiple of $d$ and $d^{\prime}$. We suppose that $\mathbb{K}$ contains a primitive $\ell$-th root of unity. We will construct a finite-dimensional Hopf algebra $\mathfrak{u}_{r, s}\left(G_{2}\right)$ of dimension $\ell^{16}$ as a quotient of $U_{r, s}\left(G_{2}\right)$ by a Hopf ideal $\mathscr{I}$ that is generated by certain central elements.

Commutation relations in $\boldsymbol{U}^{+}$and central elements. We give some commutation relations that hold in the positive part of 2-parameter quantum group of type $G_{2}$; the relations are useful for determining central elements in this subsection and integrals in Section 6.

In the following lemmas, we adopt the following notations: $\xi=r^{2}-s^{2}+r s$, $\eta=r^{2}-s^{2}-r s$ and $\zeta=\left(r^{3}-s^{3}\right)(r+s)^{-1}$, and we will need the $r$, $s$-integers, 
factorials and binomial coefficients defined for positive integers $i, n$ and $m$ by

$$
\begin{aligned}
{[n]_{i}:=\frac{r^{i n}-s^{i n}}{r^{i}-s^{i}}, } & {[n]_{1}:=[n], } \\
{[n] !:=[n][n-1] \cdots[2][1], } & {\left[\begin{array}{c}
n \\
m
\end{array}\right]:=\frac{[n] !}{[m] ![n-m] !} . }
\end{aligned}
$$

By convention, $[0]=0$ and $[0] !=1$.

Lemma 3.1. The following relations hold in $U^{+}$:

$$
\begin{aligned}
E_{112} e_{2} & =(r s)^{3} e_{2} E_{112}+r\left(r^{2}-s^{2}\right) E_{12}^{2} . \\
E_{11212} e_{2} & =\left(r^{2} s\right)^{3} e_{2} E_{11212}+r^{3}(r-s)\left(r^{2}-s^{2}\right) E_{12}^{3} . \\
E_{1112} e_{2} & =\left(r s^{2}\right)^{3} e_{2} E_{1112}+\left(r^{2} s\right)\left(r^{3}-s^{3}\right) E_{12} E_{112}+r \eta E_{11212} . \\
E_{1112} E_{12} & =(r s)^{3} E_{12} E_{1112}+r \zeta E_{112}^{2} . \\
e_{1} E_{11212} & =(r s)^{3} E_{11212} e_{1}+r \zeta E_{112}^{2} . \\
E_{1112} E_{112} & =r^{3} E_{112} E_{1112} \cdot{ }^{1} \\
E_{1112} E_{11212} & =\left(r^{2} s\right)^{3} E_{11212} E_{1112}+r^{3} \zeta(r-s) E_{112}^{3} . \\
E_{11212} E_{12} & =r^{3} E_{12} E_{11212} . \\
E_{112} E_{11212} & =r^{3} E_{11212} E_{112} .
\end{aligned}
$$

Proof. Part (1) follows directly from (2-3), (2-6) and (2-2).

Part (2) follows from (2-5), (2-6) and (1).

Part (3): Using (2-3), we have

$$
e_{1} E_{12}^{2}=E_{112} E_{12}+r s^{2} E_{12} E_{112}+\left(r s^{2}\right)^{2} E_{12}^{2} e_{1} .
$$

Also, using (2-4), (1) and (2-2), (2-5), we have

$$
\begin{aligned}
E_{1112} e_{2}= & \left(e_{1} E_{112}-r^{2} s E_{112} e_{1}\right) e_{2} \\
= & (r s)^{3} e_{1} e_{2} E_{112}+r\left(r^{2}-s^{2}\right) e_{1} E_{12}^{2}-r^{2} s E_{112} e_{1} e_{2} \\
= & (r s)^{3}\left(E_{12}+s^{3} e_{2} e_{1}\right) E_{112}+r\left(r^{2}-s^{2}\right) e_{1} E_{12}^{2}-r^{2} s E_{112}\left(E_{12}+s^{3} e_{2} e_{1}\right) \\
= & (r s)^{3} E_{12} E_{112}+\left(r s^{2}\right)^{3} e_{2} e_{1} E_{112}+r\left(r^{2}-s^{2}\right) e_{1} E_{12}^{2}-r^{2} s E_{112} E_{12} \\
& \quad-\left(r s^{2}\right)^{2}\left((r s)^{3} e_{2} E_{112}+r\left(r^{2}-s^{2}\right) E_{12}^{2}\right) e_{1} \\
= & (r s)^{3} E_{12} E_{112}+\left(r s^{2}\right)^{3} e_{2} e_{1} E_{112}+r\left(r^{2}-s^{2}\right) E_{112} E_{12} \\
& \quad+(r s)^{2}\left(r^{2}-s^{2}\right) E_{12} E_{112}+r^{3} s^{4}\left(r^{2}-s^{2}\right) E_{12}^{2} e_{1}-r^{2} s E_{112} E_{12} \\
& \quad+\left(r s^{2}\right)^{3} e_{2} E_{1112}-\left(r s^{2}\right)^{3} e_{2} e_{1} E_{112}-r^{3} s^{4}\left(r^{2}-s^{2}\right) E_{12}^{2} e_{1} \\
& \quad\left(r s^{2}\right)^{3} e_{2} E_{1112}+\left(r^{2} s\right)\left(r^{3}-s^{3}\right) E_{12} E_{112}+r \eta E_{11212 .}
\end{aligned}
$$


Part (4): Using (2-2), (2-7) and (3), we have

$$
\begin{aligned}
E_{1112} E_{12}= & E_{1112}\left(e_{1} e_{2}-s^{3} e_{2} e_{1}\right) \\
= & r^{-3} e_{1} E_{1112} e_{2}-s^{3} E_{1112} e_{2} e_{1} \\
= & r^{-3} e_{1}\left(\left(r s^{2}\right)^{3} e_{2} E_{1112}+(r s)^{2} \xi E_{12} E_{112}+r \eta E_{112} E_{12}\right)
\end{aligned}
$$

that is, $(r+s) E_{1112} E_{12}=(r s)^{3}(r+s) E_{12} E_{1112}+r\left(r^{3}-s^{3}\right) E_{112}^{2}$. Since we have assumed that $r^{2} \neq s^{2}$, this implies (4).

Part (5): Using (2-5), (2-4) and (2-3), we have

$$
e_{1} E_{11212}-(r s)^{3} E_{11212} e_{1}=E_{1112} E_{12}-(r s)^{3} E_{12} E_{1112},
$$

and using (4), we have the desired result.

Part (6): Using (2-3), (2-7) and (4), we have

$$
E_{1112} E_{112}=s^{3} E_{112} E_{1112}+r^{-2} \zeta\left(e_{1} E_{112}^{2}-\left(r^{2} s\right)^{2} E_{112}^{2} e_{1}\right),
$$

on the other hand, using (2-4), we have

$$
E_{1112} E_{112}=e_{1} E_{112}^{2}-r^{2} s E_{112} E_{1112}-\left(r^{2} s\right)^{2} E_{112}^{2} e_{1},
$$

so that, because $r^{2}+s^{2} \neq 0$, we get $E_{1112} E_{112}=r^{3} E_{112} E_{1112}$.

Part (7) can be proved by using (2-5), (6) and (4).

Part (8): It is easy to check these relations:

$$
\begin{aligned}
e_{1} E_{12}^{3}-\left(r s^{2}\right)^{3} E_{12}^{3} e_{1} & =E_{112} E_{12}^{2}+r s^{2} E_{12} E_{11212}+r^{2} s^{3}(r+s) E_{12}^{2} E_{112}, \\
E_{112}^{2} e_{2}-(r s)^{6} e_{2} E_{112}^{2} & =r^{4} s^{3}\left(r^{2}-s^{2}\right) E_{12}^{2} E_{112}+r\left(r^{2}-s^{2}\right) E_{112} E_{12}^{2}, \\
E_{112} E_{12}^{2}-\left(r^{2} s\right)^{2} E_{12}^{2} E_{112} & =E_{11212} E_{12}+r^{2} s E_{12} E_{11212} .
\end{aligned}
$$

Using (2-2), (5) and (2), we have

$$
\begin{aligned}
E_{11212} E_{12}= & E_{11212}\left(e_{1} e_{2}-s^{3} e_{2} e_{1}\right) \\
= & (r s)^{-3}\left(e_{1} E_{11212}-r \zeta E_{112}^{2}\right) e_{2} \\
& \quad-s^{3}\left(\left(r^{2} s\right)^{3} e_{2} E_{11212}+r^{3}(r-s)\left(r^{2}-s^{2}\right) E_{12}^{3}\right) e_{1}
\end{aligned}
$$




$$
\begin{aligned}
= & s^{-3} e_{1}\left((r s)^{3} e_{2} E_{11212}+(r-s)\left(r^{2}-s^{2}\right) E_{12}^{3}\right)-r^{-2} s^{-3} \zeta E_{112}^{2} e_{2} \\
& \quad-(r s)^{3} e_{2}\left(e_{1} E_{11212}-r \zeta E_{112}^{2}\right)-(r s)^{3}(r-s)\left(r^{2}-s^{2}\right) E_{12}^{3} e_{1} \\
= & r^{3} E_{12} E_{11212} \\
& +s^{-3}(r-s)\left(r^{2}-s^{2}\right)\left(E_{112} E_{12}^{2}+r s^{2} E_{12} E_{11212}+r^{2} s^{3}(r+s) E_{12}^{2} E_{112}\right) \\
& -r^{-2} s^{-3} \zeta\left(r^{4} s^{3}\left(r^{2}-s^{2}\right) E_{12}^{2} E_{112}+r\left(r^{2}-s^{2}\right) E_{112} E_{12}^{2}\right) \\
= & r^{3} E_{12} E_{11212}-(r s)^{-1}(r-s)^{2}\left(E_{112} E_{12}^{2}-\left(r^{2} s\right)^{2} E_{12}^{2} E_{112}\right) \\
& \quad+r s^{-1}(r-s)\left(r^{2}-s^{2}\right) E_{12} E_{11212} \\
= & r^{3} E_{12} E_{11212}-(r s)^{-1}(r-s)^{2}\left(E_{11212} E_{12}+r^{2} s E_{12} E_{11212}\right) \\
& +r s^{-1}(r-s)\left(r^{2}-s^{2}\right) E_{12} E_{11212 .}
\end{aligned}
$$

Thus,

$$
\left(1+r^{-1} s^{-1}(r-s)^{2}\right) E_{11212} E_{12}=\left(r^{3}+r s^{-1}(r-s)\left(r^{2}-s^{2}\right)-r(r-s)^{2}\right) E_{12} E_{11212} .
$$

Since we have assumed that $r^{2}+s^{2}-r s \neq 0$, this implies $E_{11212} E_{12}=r^{3} E_{12} E_{11212}$.

Part (9): On one hand, we have

$$
\begin{aligned}
& E_{112} E_{11212}=\left(e_{1} E_{12}-r s^{2} E_{12} e_{1}\right) E_{11212} \\
& =r^{-3} e_{1} E_{11212} E_{12}-r s^{2} E_{12} e_{1} E_{11212} \\
& =r^{-3}\left((r s)^{3} E_{11212} e_{1}+r \zeta E_{112}^{2}\right) E_{12}-r s^{2} E_{12}\left((r s)^{3} E_{11212} e_{1}+r \zeta E_{112}^{2}\right) \\
& =s^{3} E_{11212} E_{112}+r^{-2} \zeta\left(E_{112}^{2} E_{12}-\left(r^{2} s\right)^{2} E_{12} E_{112}^{2}\right) .
\end{aligned}
$$

On the other hand,

$$
E_{112}^{2} E_{12}=E_{112} E_{11212}+r^{2} s E_{11212} E_{112}+\left(r^{2} s\right)^{2} E_{12} E_{112}^{2} .
$$

Hence we obtain $E_{112} E_{11212}=r^{3} E_{11212} E_{112}$ since $r^{2}+s^{2} \neq 0$.

The main result of this subsection is the following theorem.

Theorem 3.2. The elements $E_{\alpha}^{\ell}, F_{\alpha}^{\ell}, \omega_{k}^{\ell}-1$ and $\left(\omega_{k}^{\prime}\right)^{\ell}-1$ are central in $U_{r, s}\left(G_{2}\right)$ for $\alpha \in \Phi^{+}$and $k=1,2$.

Theorem 3.2 will be proved through a sequence of lemmas.

Lemma 3.3. Let $x, y, z$ be elements of a $\mathbb{K}$-algebra such that $y x=\alpha x y+z$ for some $\alpha \in \mathbb{K}$, and let $n$ be a natural number.

(1) If $z x=\beta x z$ for some $\beta \in \mathbb{K}$ not equal to $\alpha$, then

$$
y x^{n}=\alpha^{n} x^{n} y+\frac{\alpha^{n}-\beta^{n}}{\alpha-\beta} x^{n-1} z
$$


(2) If $y z=\beta z y$ for some $\beta \in \mathbb{K}$ not equal to $\alpha$, then

$$
y^{n} x=\alpha^{n} x y^{n}+\frac{\alpha^{n}-\beta^{n}}{\alpha-\beta} z y^{n-1} .
$$

Lemma 3.4. For any positive integer $a$, the following equalities hold.

$$
\begin{aligned}
E_{12}^{a} e_{2} & =r^{3 a} e_{2} E_{12}^{a} . \\
e_{1} E_{1112}^{a} & =r^{3 a} E_{1112}^{a} e_{1} . \\
e_{1} E_{112}^{a} & =\left(r^{2} s\right)^{a} E_{112}^{a} e_{1}+r^{2(a-1)}[a] E_{112}^{a-1} E_{1112} . \\
e_{1} E_{11212}^{a} & =(r s)^{3 a} E_{11212}^{a} e_{1}+r^{3 a-2} \zeta[a]_{3} E_{11212}^{a-1} E_{112}^{2} . \\
E_{11212}^{a} e_{2} & =\left(r^{2} s\right)^{3 a} e_{2} E_{11212}^{a}+r^{3(2 a-1)}(r-s)\left(r^{2}-s^{2}\right)[a]_{3} E_{12}^{3} E_{11212}^{a-1} . \\
e_{1} e_{2}^{a} & =s^{3 a} e_{2}^{a} e_{1}+[a]_{3} e_{2}^{a-1} E_{12} .
\end{aligned}
$$

Proof. Parts (1) and (2) follow from (2-6) and (2-7).

Part (3) follows from Lemma 3.3(1), where $x=E_{112}, y=e_{1}, z=E_{1112}, \alpha=r^{2} s$, $\beta=r^{3}$, and $e_{1} E_{112}=E_{1112}+r^{2} s E_{112} e_{1}$ and $E_{1112} E_{112}=r^{3} E_{112} E_{1112}$.

Part (4) follows from Lemma 3.3(1) and Lemma 3.1(5) and (9), where $x=E_{11212}$, $y=e_{1}, \quad z=r \zeta E_{112}^{2}, \alpha=(r s)^{3}, \beta=r^{6}$, and $e_{1} E_{11212}=(r s)^{3} E_{11212} e_{1}+r \zeta E_{112}^{2}$ and $E_{112} E_{11212}=r^{3} E_{11212} E_{112}$.

Part (5) follows from Lemma 3.1(2) and Lemma 3.3(2), where $y=E_{11212}, x=e_{2}$, $z=r^{3}(r-s)\left(r^{2}-s^{2}\right) E_{12}^{3}, \alpha=\left(r^{2} s\right)^{3}$ and $\beta=r^{9}$.

Part (6) follows from Lemma 3.3(1), together with (2-2) and (2-6).

Lemma 3.5. The following equalities hold for any positive integer $a$,

$$
\begin{aligned}
& E_{112}^{a} e_{2}=(r s)^{3 a} e_{2} E_{112}^{a}+r^{3(a-2)}\left(r^{2}-s^{2}\right) \\
& \cdot\left(r^{4} s^{2(a-1)}[a] E_{12}^{2} E_{112}^{2}+r^{3} s^{a-2}[2]\left[\begin{array}{l}
a \\
2
\end{array}\right]\right. \\
& E_{12} E_{11212} E_{112} \\
& \left.+[2]\left[\begin{array}{c}
a \\
3
\end{array}\right] E_{11212}^{2}\right) E_{112}^{a-3} .
\end{aligned}
$$

$$
\begin{gathered}
e_{1}^{a} e_{2}=s^{3 a} e_{2} e_{1}^{a}+s^{2(a-1)}[a] E_{12} e_{1}^{a-1}+s^{a-2}\left[\begin{array}{l}
a \\
2
\end{array}\right] E_{112} e_{1}^{a-2}+\left[\begin{array}{l}
a \\
3
\end{array}\right] E_{1112} e_{1}^{a-3} \\
\text { for } a>4 \\
e_{1} E_{12}^{a}=\left(r s^{2}\right)^{a} E_{12}^{a} e_{1}+(r s)^{a-1}[a] E_{12}^{a-1} E_{112}+r^{a-2}\left[\begin{array}{l}
a \\
2
\end{array}\right] E_{12}^{a-2} E_{11212} \\
E_{1112}^{a} e_{2}=\left(r s^{2}\right)^{3 a} e_{2} E_{1112}^{a}+\left(r^{2} s\right)(r s)^{3(a-1)}\left(r^{3}-s^{3}\right)[a]_{3} E_{12} E_{112} E_{1112}^{a-1} \\
+r \eta(r s)^{3(a-1)}[a]_{3} E_{11212} E_{1112}^{a-1} \\
+r^{3(a-1)} \zeta(r-s)[2]_{3}\left[\begin{array}{l}
a \\
2
\end{array}\right]_{3} E_{112}^{3} E_{112}^{a-2}
\end{gathered}
$$

Proof. Part (1): Use induction on $a$. If $a=1$, this is Lemma 3.1(1). Using the equation $E_{112} E_{12}^{2}=\left(r^{2} s\right)^{2} E_{12}^{2} E_{112}+r^{2}[2] E_{12} E_{11212}$, (2-5) and Lemma 3.1(9), we 
see (1) is true for $a=2,3,4$. Supposing that (1) is true for all $a \geq 4$, we obtain

$$
\begin{aligned}
& E_{112}^{a+1} e_{2}=(r s)^{3 a} E_{112} e_{2} E_{112}^{a} \\
& +r^{3(a-2)}\left(r^{2}-s^{2}\right)\left(r^{4} s^{2(a-1)}[a] E_{112} E_{12}^{2} E_{112}^{2}\right. \\
& +r^{3} s^{a-2}[2]\left[\begin{array}{l}
a \\
2
\end{array}\right] E_{112} E_{12} E_{11212} E_{112} \\
& \left.+[2]\left[\begin{array}{c}
a \\
3
\end{array}\right] E_{112} E_{11212}^{2}\right) E_{112}^{a-3} \\
& =(r s)^{3(a+1)} e_{2} E_{112}^{a+1}+r(r s)^{3 a}\left(r^{2}-s^{2}\right) E_{12}^{2} E_{112}^{a} \\
& +r^{3(a-2)}\left(r^{2}-s^{2}\right)\left(r^{8} s^{2 a}[a] E_{12}^{2} E_{112}^{3}+r^{6} s^{2 a-2}[2][a] E_{12} E_{11212} E_{112}^{2}\right. \\
& +r^{3} s^{a-2}[2]\left[\begin{array}{c}
a \\
2
\end{array}\right] E_{11212}^{2} E_{112} \\
& \left.+r^{8} s^{a-1}[2]\left[\begin{array}{l}
a \\
2
\end{array}\right] E_{12} E_{11212} E_{112}^{2}+r^{6}[2]\left[\begin{array}{l}
a \\
3
\end{array}\right] E_{11212}^{2} E_{112}\right) E_{112}^{a-3} \\
& =(r s)^{3(a+1)} e_{2} E_{112}^{a+1} \\
& +r^{3(a-1)}\left(r^{2}-s^{2}\right)\left(r^{4} s^{2 a}[a+1] E_{12}^{2} E_{112}^{2}\right. \\
& +r^{3} s^{a-1}[2]\left[\begin{array}{c}
a+1 \\
2
\end{array}\right] E_{12} E_{11212} E_{112} \\
& \left.+[2]\left[\begin{array}{c}
a+1 \\
3
\end{array}\right] E_{11212}^{2}\right) E_{112}^{a-2}
\end{aligned}
$$

by the induction hypothesis.

Part (2): We use induction on $a$. If $a=4$, using the defining relations (2-2), (2-3), (2-4), and (2-7), and a simple computation, we have

$$
e_{1}^{4} e_{2}=[4] E_{1112} e_{1}+s^{2}\left[\begin{array}{l}
4 \\
2
\end{array}\right] E_{112} e_{1}^{2}+s^{6}[4] E_{12} e_{1}^{3}+s^{12} e_{2} e_{1}^{4} .
$$

Furthermore, using the induction hypothesis and (2-7), we obtain $e_{1}^{a+1} e_{2}=\left[\begin{array}{c}a+1 \\ 3\end{array}\right] E_{1112} e_{1}^{a-2}+s^{a-1}\left[\begin{array}{c}a+1 \\ 2\end{array}\right] E_{112} e_{1}^{a-1}+s^{2 a}[a+1] E_{12} e_{1}^{a}+s^{3(a+1)} e_{2} e_{1}^{a+1}$.

Part (3): We use induction on $a$. If $a=2$, using (2-3), we have

$$
e_{1} E_{12}^{2}=E_{11212}+r s[2] E_{12} E_{112}+\left(r s^{2}\right)^{2} E_{12}^{2} e_{1} .
$$

Suppose that (3) is true for $a \geq 2$. Then, using the induction hypothesis, (2-5) and Lemma 3.1(8), we obtain

$$
\begin{aligned}
e_{1} E_{12}^{a+1}= & \left(r s^{2}\right)^{a} E_{12}^{a} e_{1} E_{12}+(r s)^{a-1}[a] E_{12}^{a-1} E_{112} E_{12}+r^{a-2}\left[\begin{array}{c}
a \\
2
\end{array}\right] E_{12}^{a-2} E_{11212} E_{12} \\
= & \left(r s^{2}\right)^{a} E_{12}^{a} E_{112}+\left(r s^{2}\right)^{a+1} E_{12}^{a+1} e_{1}+(r s)^{a-1}[a] E_{12}^{a-1} E_{11212} \\
& \quad+r^{a+1} s^{a}[a] E_{12}^{a} E_{112}+r^{a+1}\left[\begin{array}{c}
a \\
2
\end{array}\right] E_{12}^{a-1} E_{11212} \\
= & \left(r s^{2}\right)^{a+1} E_{12}^{a+1} e_{1}+(r s)^{a}[a+1] E_{12}^{a} E_{112}+r^{a-1}\left[\begin{array}{c}
a+1 \\
2
\end{array}\right] E_{12}^{a-1} E_{11212} .
\end{aligned}
$$


Part (4): We use induction on $a$. If $a=1$, this is the relation in Lemma 3.1(3). Suppose that (4) is true for all $a \geq 1$. Using the induction hypothesis and Lemma 3.1(3), (4), (6) and (7), we obtain

$$
\begin{aligned}
& E_{1112}^{a+1} e_{2}=\left(r s^{2}\right)^{3 a}\left(E_{1112} e_{2}\right) E_{1112}^{a} \\
& +\left(r^{2} s\right)(r s)^{3(a-1)}\left(r^{3}-s^{3}\right)[a]_{3}\left(E_{1112} E_{12}\right) E_{112} E_{1112}^{a-1} \\
& +r \eta(r s)^{3(a-1)}[a]_{3}\left(E_{1112} E_{11212}\right) E_{1112}^{a-1} \\
& +r^{3(a-1)} \zeta(r-s)[2]_{3}\left[\begin{array}{l}
a \\
2
\end{array}\right]_{3}\left(E_{1112} E_{112}^{3}\right) E_{1112}^{a-2} \\
& =\left(r s^{2}\right)^{3 a}\left(\left(r s^{2}\right)^{3} e_{2} E_{1112}+\left(r^{2} s\right)\left(r^{3}-s^{3}\right) E_{12} E_{112}+r \eta E_{11212}\right) E_{1112}^{a} \\
& +\left(r^{2} s\right)(r s)^{3(a-1)}\left(r^{3}-s^{3}\right)[a]_{3}\left((r s)^{3} E_{12} E_{1112}+r \zeta E_{112}^{2}\right) E_{112} E_{1112}^{a-1} \\
& +r \eta(r s)^{3(a-1)}[a]_{3}\left(\left(r^{2} s\right)^{3} E_{11212} E_{1112}+r^{3} \zeta(r-s) E_{112}^{3}\right) E_{1112}^{a-1} \\
& +r^{3 a+6} \zeta(r-s)[2]_{3}\left[\begin{array}{l}
a \\
2
\end{array}\right]_{3} E_{112}^{3} E_{1112}^{a-1} \\
& =\left(r s^{2}\right)^{3(a+1)} e_{2} E_{1112}^{a+1}+\left(r^{2} s\right)(r s)^{3 a}\left(r^{3}-s^{3}\right)[a+1]_{3} E_{12} E_{112} E_{1112}^{a} \\
& +r \eta(r s)^{3 a}[a+1]_{3} E_{11212} E_{1112}^{a}+r^{3 a} \zeta(r-s)[2]_{3}\left[\begin{array}{c}
a+1 \\
2
\end{array}\right]_{3} E_{112}^{3} E_{1112}^{a-1} \text {. }
\end{aligned}
$$

This completes the proof.

Lemma 3.6. For any positive integer a, these equalities hold:

$$
\begin{aligned}
e_{k}^{a} f_{k} & =f_{k} e_{k}^{a}+[a]_{k} e_{k}^{a-1} \frac{s_{k}^{-a+1} \omega_{k}-r_{k}^{-a+1} \omega_{k}^{\prime}}{r_{k}-s_{k}} \text { for } 1 \leq k \leq 2 . \\
E_{12}^{a} f_{1} & =f_{1} E_{12}^{a}-r^{2(a-1)}[3][a] e_{2} E_{12}^{a-1} \omega_{1}^{\prime} . \\
E_{112}^{a} f_{1} & =f_{1} E_{112}^{a}-(r s)^{a-1}[2]^{2}[a] E_{12} E_{112}^{a-1} \omega_{1}^{\prime} \\
& \quad-r^{a-2}[2]^{2}\left[\begin{array}{c}
a \\
2
\end{array}\right] E_{11212} E_{112}^{a-2} \omega_{1}^{\prime} .
\end{aligned}
$$

Proof. Part (1) can be easily verified by induction on $a$.

Parts (2), (4) and (5) follow from Lemma 3.3(2) and the facts

$$
\begin{aligned}
E_{12} f_{1} & =f_{1} E_{12}-[3] e_{2} \omega_{1}^{\prime}, & E_{1112} f_{1} & =f_{1} E_{1112}-[3] E_{112} \omega_{1}^{\prime}, \\
E_{112} f_{1} & =f_{1} E_{112}-[2]^{2} E_{12} \omega_{1}^{\prime}, & E_{11212} f_{1} & =f_{1} E_{11212}-r\left(r^{2}-s^{2}\right)[3] E_{12}^{2} \omega_{1}^{\prime} .
\end{aligned}
$$

Part (3) can be verified by induction on $a$.

$$
\begin{aligned}
E_{112}^{a+1} f_{1}= & \left(E_{112} f_{1}\right) E_{112}^{a}-(r s)^{a-1}[2]^{2}[a]\left(E_{112} E_{12}\right) E_{112}^{a-1} \omega_{1}^{\prime} \\
& -r^{a-2}[2]^{2}\left[\begin{array}{c}
a \\
2
\end{array}\right]\left(E_{112} E_{11212}\right) E_{112}^{a-2} \omega_{1}^{\prime} \\
= & f_{1} E_{112}^{a+1}-(r s)^{a}[2]^{2}[a+1] E_{12} E_{112}^{a} \omega_{1}^{\prime}-r^{a-1}[2]^{2}\left[\begin{array}{c}
a+1 \\
2
\end{array}\right] E_{11212} E_{112}^{a-1} \omega_{1}^{\prime} .
\end{aligned}
$$

This completes the proof. 
Lemma 3.7. For any positive integer a,

(1) $E_{12}^{a} f_{2}=f_{2} E_{12}^{a}+\omega_{2} E_{12}^{a-3}\left(s^{2(a-1)} E_{12}^{2} e_{1}+s^{a-2}\left[\begin{array}{l}a \\ 2\end{array}\right] E_{12} E_{112}+\left[\begin{array}{l}a \\ 3\end{array}\right] E_{11212}\right)$;

(2) $E_{112}^{a} f_{2}=f_{2} E_{112}^{a}+r^{3(a-2)}\left(r^{2}-s^{2}\right) \omega_{2} E_{112}^{a-3}$

$$
\begin{aligned}
\times\left([2]\left[\begin{array}{l}
a \\
3
\end{array}\right] E_{1112}^{2}+r^{3} s^{a-2}[2]\left[\begin{array}{l}
a \\
2
\end{array}\right] E_{112} E_{1112} e_{1}\right. \\
\left.+[a] r^{4} s^{2(a-1)} E_{112}^{2} e_{1}^{2}\right) ;
\end{aligned}
$$

(3) $E_{1112}^{a} f_{2}=f_{2} E_{1112}^{a}+r^{-3(a-2)}\left(r^{2}-s^{2}\right)(r-s)[a]_{3} \omega_{2} e_{1}^{3} E_{1112}^{a-1}$;

(4) $E_{11212}^{a} f_{2}=f_{2} E_{11212}^{a}+r(r s)^{3(a-1)}[a]_{3} \omega_{2} E_{11212}^{a-1}\left(\eta E_{1112}+r s\left(r^{3}-s^{3}\right) E_{112} e_{1}\right)$

$$
+r^{3(a-1)} \zeta(r-s)[2]_{3}\left[\begin{array}{l}
a \\
2
\end{array}\right]_{3} \omega_{2} E_{11212}^{a-2} E_{112}^{3} .
$$

Proof. Part (1): Because $E_{12} f_{2}=f_{2} E_{12}+\omega_{2} e_{1}$, it is easy to check the cases when $a=2,3,4$. Using the induction hypothesis and Lemma 3.5(3), we have

$$
\begin{aligned}
E_{12}^{a+1} f_{2}= & E_{12} f_{2} E_{12}^{a}+\omega_{2} E_{12}^{a-2}\left(s^{2 a+1}[a] E_{12}^{2} e_{1}+s^{a+1}\left[\begin{array}{c}
a \\
2
\end{array}\right] E_{12} E_{112}+s^{3}\left[\begin{array}{c}
a \\
3
\end{array}\right] E_{11212}\right) \\
= & f_{2} E_{12}^{a+1}+r^{a-2} \omega_{2}\left(r^{2} s^{2 a} E_{12}^{a} e_{1}+r s^{a-1}[a] E_{12}^{a-1} E_{112}+\left[\begin{array}{c}
a \\
2
\end{array}\right] E_{12}^{a-2} E_{11212}\right) \\
& \quad+\omega_{2} E_{12}^{a-2}\left(s^{2 a+1}[a] E_{12}^{2} e_{1}+s^{a+1}\left[\begin{array}{c}
a \\
2
\end{array}\right] E_{12} E_{112}+s^{3}\left[\begin{array}{c}
a \\
3
\end{array}\right] E_{11212}\right) \\
= & f_{2} E_{12}^{a+1}+\omega_{2} E_{12}^{a-2}\left(s^{2 a}[a+1] E_{12}^{2} e_{1}+s^{a-1}\left[\begin{array}{c}
a+1 \\
2
\end{array}\right] E_{12} E_{112}+\left[\begin{array}{c}
a+1 \\
3
\end{array}\right] E_{11212}\right) .
\end{aligned}
$$

Part (2): From (1), it is easy to calculate $E_{112} f_{2}=f_{2} E_{112}+r\left(r^{2}-s^{2}\right) \omega_{2} e_{1}^{2}$. The relation below is obtained by Lemma 3.4(3):

$$
e_{1}^{2} E_{112}^{a}=r^{4 a-6} E_{112}^{a-2}\left([2]\left[\begin{array}{l}
a \\
2
\end{array}\right] E_{1112}^{2}+r^{4} s^{a-1}[2][a] E_{112} E_{1112} e_{1}+r^{6} s^{2 a} E_{112}^{2} e_{1}^{2}\right) .
$$

For $a \geq 1$, we have by induction on $a$

$$
\begin{gathered}
E_{112}^{a+1} f_{2}=\left(E_{112} f_{2}\right) E_{112}^{a}+r^{3(a-1)} s^{3}\left(r^{2}-s^{2}\right) \omega_{2} E_{112}^{a-2} \\
\times\left([2]\left[\begin{array}{c}
a \\
3
\end{array}\right] E_{1112}^{2}+r^{3} s^{a-2}[2]\left[\begin{array}{l}
a \\
2
\end{array}\right] E_{112} E_{1112} e_{1}\right. \\
\left.+r^{4} s^{2(a-1)}[a] E_{112}^{2} e_{1}^{2}\right) \\
=f_{2} E_{112}^{a+1}+r\left(r^{2}-s^{2}\right) r^{4 a-6} \omega_{2} E_{112}^{a-2} \\
\times\left([2]\left[\begin{array}{c}
a \\
2
\end{array}\right] E_{1112}^{2}+r^{4} s^{a-1}[2][a] E_{112} E_{1112} e_{1}+r^{6} s^{2 a} E_{112}^{2} e_{1}^{2}\right) \\
+r^{3(a-1)} s^{3}\left(r^{2}-s^{2}\right) \omega_{2} E_{112}^{a-2} \\
\quad \times\left([2]\left[\begin{array}{c}
a \\
3
\end{array}\right] E_{1112}^{2}+r^{3} s^{a-2}[2]\left[\begin{array}{c}
a \\
2
\end{array}\right] E_{112} E_{1112} e_{1}+r^{4} s^{2(a-1)}[a] E_{112}^{2} e_{1}^{2}\right) \\
=f_{2} E_{112}^{a+1}+r^{3(a-1)}\left(r^{2}-s^{2}\right) \omega_{2} E_{112}^{a-2} \\
\quad \times\left([2]\left[\begin{array}{c}
a+1 \\
3
\end{array}\right] E_{1112}^{2}+r^{3} s^{a-1}[2]\left[\begin{array}{c}
a+1 \\
2
\end{array}\right] E_{112} E_{1112} e_{1}+r^{4} s^{2 a}[a+1] E_{112}^{2} e_{1}^{2}\right) .
\end{gathered}
$$


Part (3): We consider the case for $a=1$. It follows from (2) that

$$
E_{1112} f_{2}=f_{2} E_{1112}+r^{3}\left(r^{2}-s^{2}\right)(r-s) \omega_{2} e_{1}^{3} .
$$

So by this and Lemma 3.3(2), we can easily get (3).

Part (4): If $a=1$, we have

$$
E_{11212} f_{2}=f_{2} E_{11212}+r \eta \omega_{2} E_{1112}+r^{2} s\left(r^{3}-s^{3}\right) \omega_{2} E_{112} e_{1}
$$

by using (1) and (2). Then

$$
E_{1112} E_{11212}^{a}=\left(r^{2} s\right)^{3 a} E_{11212}^{a} E_{1112}+r^{3(2 a-1)} \zeta(r-s)[a]_{3} E_{11212}^{a-1} E_{112}^{3} .
$$

holds by Lemma 3.3(1). So (4) can be derived easily from this and Lemma 3.4(4) by induction on $a$, which completes the proof.

Proof of Theorem 3.2.. We know from Lemmas 3.4, 3.5, 3.6 and 3.7 that the elements $E_{1}^{\ell}, E_{1112}^{\ell}, E_{112}^{\ell}, E_{11212}^{\ell}, E_{12}^{\ell}$ and $E_{2}^{\ell}$ are central in $U_{r, s}\left(G_{2}\right)$. Applying $\tau$ to $E_{\alpha}$ for $\alpha \in \Phi$, we see that $F_{1}^{\ell}, F_{1112}^{\ell}, F_{112}^{\ell}, F_{11212}^{\ell}, F_{12}^{\ell}$ and $F_{2}^{\ell}$ are also central. It is easy to see that $\omega_{k}^{\ell}-1$ and $\left(\omega_{k}^{\prime}\right)^{\ell}-1$ are central too for $k=1,2$.

Remark 3.8. If $\ell=3 \ell^{\prime}$, then the elements $E_{1112}^{\ell^{\prime}}, F_{1112}^{\ell^{\prime}}, \omega_{k}^{\ell^{\prime}}-1$ and $\left(\omega_{k}^{\prime}\right)^{\ell^{\prime}}-1$ are central in $U_{r, s}\left(G_{2}\right)$ for $k=1,2$.

Restricted 2-parameter quantum groups. From now on we will assume that $\ell$ is coprime to 3.

Definition 3.9. The restricted 2-parameter quantum group is the quotient

$$
\mathfrak{u}_{r, s}\left(G_{2}\right):=U_{r, s}\left(G_{2}\right) / \mathscr{\Phi}
$$

where $\Phi$ denotes the ideal of $U_{r, s}\left(G_{2}\right)$ generated by all elements $E_{\alpha}^{\ell}$ and $F_{\alpha}^{\ell}$ for $\alpha \in \Phi^{+}$and $\omega_{k}^{\ell}-1$ and $\left(\omega_{k}^{\prime}\right)^{\ell}-1$ for $1 \leq k \leq 2$.

By Theorem 2.4 and Corollary 2.5, $\mathfrak{u}_{r, s}\left(G_{2}\right)$ is an algebra of dimension $\ell^{16}$ with linear basis

$$
E_{2}^{c_{1}} E_{12}^{c_{2}} E_{11212}^{c_{3}} E_{112}^{c_{4}} E_{1112}^{c_{5}} E_{1}^{c_{6}} \omega_{1}^{b_{1}} \omega_{2}^{b_{2}}\left(\omega_{1}^{\prime}\right)^{b_{1}^{\prime}}\left(\omega_{2}^{\prime}\right)^{b_{2}^{\prime}} F_{1}^{c_{1}^{\prime}} F_{1112}^{c_{2}^{\prime}} F_{112}^{c_{3}^{\prime}} F_{11212}^{c_{4}^{\prime}} F_{12}^{c_{5}^{\prime}} F_{2}^{c_{6}^{\prime}}
$$

where all powers range between 0 and $\ell-1$.

The remainder of this section is devoted to proving that $\mathfrak{u}_{r, s}\left(G_{2}\right)$ is a finitedimensional Hopf algebra.

First note that the generators of $\mathscr{I}$ are contained in the kernel of the counit $\varepsilon$, and so $\mathscr{I}$ is as well. Because the coproduct $\Delta$ is an algebra homomorphism and because the antipode $S$ is an algebra antihomomorphism, it suffices to show that 
$\Delta(x) \in \mathscr{I} \otimes U+U \otimes \mathscr{I}$ and $S(x) \in \mathscr{I}$ for each generator $x \in \mathscr{I}$. To accomplish this, we rely on the computations

$$
\begin{aligned}
\Delta\left(\omega_{k}^{\ell}-1\right) & =\omega_{k}^{\ell} \otimes \omega_{k}^{\ell}-1 \otimes 1 \\
& =\left(\omega_{k}^{\ell}-1\right) \otimes \omega_{k}^{\ell}+1 \otimes\left(\omega_{k}^{\ell}-1\right) \in \mathscr{I} \otimes U+U \otimes \mathscr{I},
\end{aligned}
$$

and $S\left(\omega_{k}^{\ell}-1\right)=-\omega_{k}^{-\ell}\left(\omega_{k}^{\ell}-1\right) \in \mathscr{I}$. The argument for $\left(\omega_{k}^{\prime}\right)^{\ell}-1$ is similar. In determining $\Delta(x)$ for each generator $x \in \mathscr{I}$, we adopt the notation

$$
\omega_{12}=\omega_{1} \omega_{2}, \quad \omega_{112}=\omega_{1}^{2} \omega_{2}, \quad \omega_{1112}=\omega_{1}^{3} \omega_{2}, \quad \omega_{11212}=\omega_{1}^{3} \omega_{2}^{2} .
$$

Some simple computations give rise to these facts:

\section{Lemma 3.10.}

$$
\Delta\left(E_{12}\right)=E_{12} \otimes 1+\omega_{12} \otimes E_{12}+\left(r^{3}-s^{3}\right) \omega_{2} e_{1} \otimes e_{2} ;
$$$$
\Delta\left(E_{112}\right)=E_{112} \otimes 1+\omega_{112} \otimes E_{112}+(r+s)\left(r^{2}-s^{2}\right) \omega_{12} e_{1} \otimes E_{12}
$$

$$
+r\left(r^{2}-s^{2}\right) \times\left(r^{3}-s^{3}\right) \omega_{2} e_{1}^{2} \otimes e_{2} ;
$$

(3) $\Delta\left(E_{1112}\right)=E_{1112} \otimes 1+\omega_{1112} \otimes E_{1112}+\left(r^{3}-s^{3}\right) \omega_{112} e_{1} \otimes E_{112}$

$$
\begin{aligned}
& +r\left(r^{2}-s^{2}\right) \times\left(r^{3}-s^{3}\right) \omega_{12} e_{1}^{2} \otimes E_{12} \\
& +r^{3}(r-s)\left(r^{2}-s^{2}\right)\left(r^{3}-s^{3}\right) \omega_{2} e_{1}^{3} \otimes e_{2} ;
\end{aligned}
$$

(4) $\Delta\left(E_{11212}\right)=E_{11212} \otimes 1+\omega_{11212} \otimes E_{11212}+r\left(r^{2}-s^{2}\right)\left(r^{3}-s^{3}\right) \omega_{12}^{2} e_{1} \otimes E_{12}^{2}$

$$
\begin{aligned}
& \quad+r\left(r^{2}-s^{2}\right)\left(r^{3}-s^{3}\right)^{2} \omega_{12} \omega_{2} e_{1}^{2} \otimes E_{12} e_{2} \\
& +\left(r^{3}-s^{3}\right) \omega_{12} E_{112} \otimes E_{12} \\
& +r^{6}(r-s)\left(r^{2}-s^{2}\right) \times\left(r^{3}-s^{3}\right)^{2} \omega_{2}^{2} e_{1}^{3} \otimes e_{2}^{2} \\
& +r\left(r^{3}-s^{3}\right) \omega_{2}\left(\left(r^{2}-s^{2}-r s\right) E_{1112}+r s\left(r^{3}-s^{3}\right) E_{112} e_{1}\right) \otimes e_{2} .
\end{aligned}
$$

Lemma 3.11. $\Delta\left(E_{\alpha}^{\ell}\right) \in \mathscr{I} \otimes U+U \otimes \mathscr{I}$ for $\alpha \in \Phi^{+}$.

Proof. Note that

$$
\Delta\left(e_{i}^{n}\right)=\sum_{j=0}^{n}\left(\begin{array}{c}
n \\
j
\end{array}\right)_{r_{i} s_{i}^{-1}} e_{i}^{n-j} \omega_{i}^{j} \otimes e_{i}^{j}
$$

has an $r_{i} s_{i}^{-1}$-binomial expansion ${ }^{2}$ since $\left(\omega_{i} \otimes e_{i}\right)\left(e_{i} \otimes 1\right)=r_{i} s_{i}^{-1}\left(e_{i} \otimes 1\right)\left(\omega_{i} \otimes e_{i}\right)$. So we have $\Delta\left(e_{i}^{\ell}\right)=e_{i}^{\ell} \otimes 1+\omega_{i}^{\ell} \otimes e_{i}^{\ell} \in \mathscr{I} \otimes U+U \otimes \mathscr{I}$, since $r$ and $s$ are primitive $\ell$-th roots of unity, and $\left(\begin{array}{l}\ell \\ j\end{array}\right)_{r_{i} s_{i}^{-1}}=0$ for $0<j<\ell$.
${ }^{2}$ We set

$$
(n):=(n)_{r s^{-1}}:=\frac{r^{n} s^{-n}-1}{r s^{-1}-1}, \quad(n) !:=(n)(n-1) \cdots(2)(1), \quad\left(\begin{array}{l}
n \\
m
\end{array}\right):=\frac{(n) !}{(m) !(n-m) !} .
$$

By convention $(0)=0$ and $(0) !=1$. 
Note that $\Delta\left(E_{12}^{\ell}\right)=\left(E_{12} \otimes 1+\left(r^{3}-s^{3}\right) \omega_{2} e_{1} \otimes e_{2}\right)^{\ell}+\omega_{12}^{\ell} \otimes E_{12}^{\ell}$, because $\left(\omega_{12} \otimes E_{12}\right)\left(E_{12} \otimes 1+\left(r^{3}-s^{3}\right) \omega_{2} e_{1} \otimes e_{2}\right)=$

$$
r s^{-1}\left(E_{12} \otimes 1+\left(r^{3}-s^{3}\right) \omega_{2} e_{1} \otimes e_{2}\right)\left(\omega_{12} \otimes E_{12}\right) .
$$

We get

$$
\Delta\left(E_{12}^{\ell}\right)=E_{12}^{\ell} \otimes 1+r^{3 \ell(\ell-1) / 2}\left(r^{3}-s^{3}\right)^{\ell} \omega_{2}^{\ell} e_{1}^{\ell} \otimes e_{2}^{\ell}+\omega_{12}^{\ell} \otimes E_{12}^{\ell} \in \mathscr{I} \otimes U+U \otimes \mathscr{I}
$$

since $r$ and $s$ are primitive $\ell$-th roots of unity, and

$$
\left(E_{12}+\omega_{2} e_{1}\right)^{\ell}=E_{12}^{\ell}+r^{3 \ell(\ell-1) / 2} \omega_{2}^{\ell} e_{1}^{\ell} .
$$

Similarly, $\Delta\left(E_{112}^{\ell}\right), \Delta\left(E_{1112}^{\ell}\right)$ and $\Delta\left(E_{11212}^{\ell}\right)$ are in $\mathscr{I} \otimes U+U \otimes \mathscr{I}$.

Applying the antipode property to $\Delta\left(E_{\alpha}^{\ell}\right)$ for $\alpha \in \Phi^{+}$, we obtain that $S\left(E_{\alpha}^{\ell}\right) \in$ $\mathscr{I} \otimes U+U \otimes \mathscr{I}$. Using $\tau$, we find that $\Delta\left(F_{\alpha}^{\ell}\right) \in \mathscr{I} \otimes U+U \otimes \mathscr{I}$ for $\alpha \in \Phi^{+}$. Hence $\mathscr{I}$ is a Hopf ideal, and $\mathfrak{u}_{r, s}\left(G_{2}\right)$ is a finite-dimensional Hopf algebra. Then:

Theorem 3.12. $I$ is a Hopf ideal of $U_{r, s}\left(G_{2}\right)$, making $\mathfrak{u}_{r, s}\left(G_{2}\right)$ a finite-dimensional Hopf algebra.

\section{Isomorphisms of $\mathfrak{u}_{r, s}\left(G_{2}\right)$}

Write $\mathfrak{u}=\mathfrak{u}_{r, s}=\mathfrak{u}_{r, s}\left(G_{2}\right)$. Let $G$ denote the group generated by $\omega_{i}$ and $\omega_{i}^{\prime}$ for $i=1,2$ in the restricted quantum group $\mathfrak{u}$. Define linear subspace $\mathfrak{a}_{k}$ of $\mathfrak{u}$ by

$$
\mathfrak{a}_{0}=\mathbb{K} G, \quad \mathfrak{a}_{1}=\mathbb{K} G+\sum_{i=1}^{2}\left(\mathbb{K} e_{i} G+\mathbb{K} f_{i} G\right), \quad \mathfrak{a}_{k}=\left(\mathfrak{a}_{1}\right)^{k} \quad \text { for } k \geq 1 .
$$

Note that $1 \in \mathfrak{a}_{0}, \quad \Delta\left(\mathfrak{a}_{0}\right) \subseteq \mathfrak{a}_{0} \otimes \mathfrak{a}_{0}, \quad \mathfrak{a}_{1}$ generates $\mathfrak{u}$ as an algebra, and that $\Delta\left(\mathfrak{a}_{1}\right) \subseteq \mathfrak{a}_{1} \otimes \mathfrak{a}_{0}+\mathfrak{a}_{0} \otimes \mathfrak{a}_{1}$. By [Montgomery 1993], $\left\{\mathfrak{a}_{k}\right\}$ is a coalgebra filtration of $\mathfrak{u}$ and $\mathfrak{u}_{0} \subseteq \mathfrak{a}_{0}$, where the coradical $\mathfrak{u}_{0}$ of $\mathfrak{u}$ is the sum of all the simple subcoalgebras of $\mathfrak{u}$. Clearly, $\mathfrak{a}_{0} \subseteq \mathfrak{u}_{0}$ as well, and so $\mathfrak{u}_{0}=\mathbb{K} G$. This implies that $\mathfrak{u}$ is pointed, that is, every simple subcoalgebra of $\mathfrak{u}$ is one-dimensional.

Let $\mathfrak{b}$ be the Hopf subalgebra of $\mathfrak{u}=\mathfrak{u}_{r, s}\left(G_{2}\right)$ generated by $e_{i}$ and $\omega_{i}^{ \pm 1}$ for $i=1,2$, and let $\mathfrak{b}^{\prime}$ be the Hopf subalgebra generated by $f_{i}$ and $\left(\omega_{i}^{\prime}\right)^{ \pm 1}$ for $i=1,2$. The same argument shows that $\mathfrak{b}$ and $\mathfrak{b}^{\prime}$ are pointed as well. Thus, we have this:

Proposition 4.1. The restricted 2-parameter quantum group $\mathfrak{u}_{r, s}\left(G_{2}\right)$ is a pointed Hopf algebra, as are its subalgebras $\mathfrak{b}$ and $\mathfrak{b}^{\prime}$.

[Montgomery 1993, Lemma 5.5.1] indicates that $\mathfrak{a}_{k} \subseteq \mathfrak{u}_{k}$ for all $k$, where $\left\{\mathfrak{u}_{k}\right\}$ is the coradical filtration of $\mathfrak{u}$ defined inductively by $\mathfrak{u}_{k}=\Delta^{-1}\left(\mathfrak{u} \times \mathfrak{u}_{k-1}+\mathfrak{u}_{0} \times \mathfrak{u}\right)$. In particular, $\mathfrak{a}_{1} \subseteq \mathfrak{u}_{1}$. By [op. cit., Theorem 5.4.1], since $\mathfrak{u}$ is pointed, $\mathfrak{u}_{1}$ is spanned by the set of group-like elements $G$ together with all the skew-primitive elements of $\mathfrak{u}$. 
We claim that under the additional hypothesis of the Lemma 4.2 below, $\mathfrak{u}_{1}=\mathfrak{a}_{1}$. That is, each skew-primitive element of $u$ is a linear combination of elements of $G$, of $e_{i} G$, and of $f_{i} G$ for $i=1,2$.

The following lemma gives a precise description of $\mathfrak{u}_{1}$.

Lemma 4.2. Assume that $r s^{-1}$ is a primitive $\ell$-th root of unity. Then

$$
\mathfrak{u}_{1}=\mathbb{K} G+\sum_{i=1}^{2}\left(\mathbb{K} e_{i} G+\mathbb{K} f_{i} G\right) .
$$

Given two group-like elements $g$ and $h$ in a Hopf algebra $H$, let $P_{g, h}(H)$ denote the set of skew-primitive elements of $H$ given by

$$
P_{g, h}(H)=\{x \in H \mid \Delta(x)=x \otimes g+h \otimes x\} .
$$

We want to compute $P_{1, \sigma}\left(\mathfrak{u}_{r, s}\right)$ and $P_{\sigma, 1}\left(\mathfrak{u}_{r, s}\right)$ for $\sigma \in G$.

Lemma 4.3. Assume that $r s^{-1}$ is a primitive $\ell$-th root of unity. Then we have

$$
\begin{aligned}
P_{1, \omega_{i}}\left(\mathfrak{u}_{r, s}\right) & =\mathbb{K}\left(1-\omega_{i}\right)+\mathbb{K} e_{i}, \quad P_{1, \omega_{i}^{\prime-1}}\left(\mathfrak{u}_{r, s}\right)=\mathbb{K}\left(1-\omega_{i}^{\prime-1}\right)+\mathbb{K} f_{i} \omega_{i}^{\prime-1}, \\
P_{1, \sigma}\left(\mathfrak{u}_{r, s}\right) & =\mathbb{K}(1-\sigma) \quad \text { if } \sigma \notin\left\{\omega_{i}, \omega_{i}^{\prime-1} \mid i=1,2\right\} ; \\
P_{\omega_{i}^{\prime}, 1}\left(\mathfrak{u}_{r, s}\right) & =\mathbb{K}\left(1-\omega_{i}^{\prime}\right)+\mathbb{K} f_{i}, \quad P_{\omega_{i}^{-1}, 1}\left(\mathfrak{u}_{r, s}\right)=\mathbb{K}\left(1-\omega_{i}^{-1}\right)+\mathbb{K} e_{i} \omega_{i}^{-1}, \\
P_{\sigma, 1}\left(\mathfrak{u}_{r, s}\right) & =\mathbb{K}(1-\sigma) \quad \text { if } \sigma \notin\left\{\omega_{i}^{\prime}, \omega_{i}^{-1} \mid i=1,2\right\} .
\end{aligned}
$$

Proof. (1): Assuming $r s^{-1}$ is a primitive $\ell$-th root of unity, we have

$$
\mathbb{K} G+\sum_{g, h \in G} P_{g, h}\left(\mathfrak{u}_{r, s}\right)=\mathbb{K} G+\sum_{i=1}^{2}\left(\mathbb{K} e_{i} G+\mathbb{K} f_{i} G\right) .
$$

Thus, any $x \in P_{1, \sigma}\left(\mathfrak{u}_{r, s}\right)$, where $\sigma \in G$, may be written as a linear combination

$$
x=\sum_{g \in G} \gamma_{g} g+\sum_{g \in G} \sum_{i=1}^{2} \alpha_{i, g} e_{i} g+\sum_{g \in G} \sum_{i=1}^{2} \beta_{i, g} f_{i} g,
$$

where $\gamma_{g}, \alpha_{i, g}$ and $\beta_{i, g}$ are scalars. Comparing $\Delta(x)=x \otimes 1+\sigma \otimes x$ with the coproduct of the right side, which is

$\sum_{g \in G} \gamma_{g} g \otimes g+\sum_{g \in G} \sum_{i=1}^{2} \alpha_{i, g}\left(e_{i} g \otimes g+\omega_{i} g \otimes e_{i} g\right)+\sum_{g \in G} \sum_{i=1}^{2} \beta_{i, g}\left(g \otimes f_{i} g+f_{i} g \otimes \omega_{i}^{\prime} g\right)$,

we obtain $\beta_{i, g}=0$ for all $i$ and $g \neq \omega_{i}^{\prime-1}$, and $\alpha_{i, g}=0$ for all $g \neq 1$. A further comparison of the group-like components yields $\gamma_{\sigma}=-\gamma_{1}$ and $\gamma_{g}=0$ for all $g \neq 1, \sigma$. Finally, comparing $\Delta(x)$ with $\Delta\left(\gamma_{1}(1-\sigma)+\sum_{i=1}^{2} \alpha_{i, 1} e_{i}+\sum_{i=1}^{2} \beta_{i, \omega_{i}^{\prime-1}} f_{i} \omega_{i}^{\prime-1}\right)$ 
yields $\alpha_{i, 1}=0$ for all $i$ when $\sigma \notin\left\{\omega_{1}, \omega_{2}\right\}$, and yields $\beta_{i, \omega_{i}^{\prime-1}}=0$ for all $i$ when $\sigma \notin\left\{\omega_{1}^{\prime-1}, \omega_{2}^{\prime-1}\right\}$.

Consider two more cases: First, when $\sigma=\omega_{i}$ for some $i$, we have $\alpha_{j, 1}=0$ for all $j \neq i$; and $\beta_{j, \omega_{j}^{\prime-1}}=0$ for all $j$. So $x=\gamma_{1}\left(1-\omega_{i}\right)+\alpha_{i, 1} e_{i}$. Second, when $\sigma=\omega_{i}^{\prime-1}$ for some $i$, we have $\beta_{j, \omega_{j}^{-1}}=0$ for all $j \neq i$, and we have $\alpha_{j, 1}=0$ for all $j$. So $x=\gamma_{1}\left(1-\omega_{i}^{\prime-1}\right)+\beta_{i, \omega_{i}^{\prime-1}} f_{i} \omega_{i}^{-1}$. Thus

$$
\begin{aligned}
P_{1, \omega_{i}}\left(\mathfrak{u}_{r, s}\right) & =\mathbb{K}\left(1-\omega_{i}\right)+\mathbb{K} e_{i} & & \text { for } 1 \leq i \leq 2, \\
P_{1, \omega_{i}^{\prime-1}}\left(\mathfrak{u}_{r, s}\right) & =\mathbb{K}\left(1-\omega_{i}^{\prime-1}\right)+\mathbb{K} f_{i} \omega_{i}^{\prime-1} & & \text { for } 1 \leq i \leq 2, \\
P_{1, \sigma}\left(\mathfrak{u}_{r, s}\right) & =\mathbb{K}(1-\sigma) & & \text { if } \sigma \notin\left\{\omega_{i}, \omega_{i}^{\prime-1}\right\} \text { for any } i .
\end{aligned}
$$

Similarly, we get (2).

Remark 4.4. The description of the set of left (right) skew-primitive elements of $\mathfrak{u}_{r, s}\left(\mathfrak{s l}_{n}\right)$ is not complete; see [Benkart and Witherspoon 2004b, formulas (3.6) and (3.7)]. This left many families of isomorphisms of $\mathfrak{u}_{r, s}\left(\mathfrak{s l}_{n}\right)$ undiscovered. In the second part of the proof of [Hu and Wang 2009, Theorem 5.4], the authors described explicitly all of the Hopf algebra isomorphisms of $\mathfrak{u}_{r, s}\left(\mathfrak{s l}_{n}\right)$; see [op. cit., Theorem 5.5].

Theorem 4.5. Assume that $r s^{-1}$ and $r^{\prime} s^{-1}$ are primitive $\ell$-th roots of unity with $\ell \neq 3,4$, and assume $\zeta$ is a 3 rd root of unity. Then $\varphi: \mathfrak{u}_{r, s} \cong \mathfrak{u}_{r^{\prime}, s^{\prime}}$ as Hopf algebras if and only if either

- $\left(r^{\prime}, s^{\prime}\right)=\zeta(r, s)$ and $\varphi$ is a diagonal isomorphism, that is,

$$
\begin{array}{rlrl}
\varphi\left(\omega_{i}\right) & =\tilde{\omega}_{i}, & & \varphi\left(\omega_{i}^{\prime}\right)=\tilde{\omega}_{i}^{\prime}, \\
\varphi\left(e_{i}\right)=a_{i} \tilde{e}_{i}, & \varphi\left(f_{i}\right)=\zeta^{\delta_{i, 1}} a_{i}^{-1} \tilde{f}_{i} \quad \text { for } a_{i} \in \mathbb{K}^{*} ; \text { or }
\end{array}
$$

- $\left(r^{\prime}, s^{\prime}\right)=\zeta(s, r)$ and

$$
\begin{aligned}
\varphi\left(\omega_{i}\right) & =\tilde{\omega}_{i}^{\prime-1}, & \varphi\left(\omega_{i}^{\prime}\right) & =\tilde{\omega}_{i}^{-1}, \\
\varphi\left(e_{i}\right) & =a_{i} \tilde{f}_{i} \tilde{\omega}_{i}^{\prime-1}, & \varphi\left(f_{i}\right) & =\zeta^{\delta_{i, 1}} a_{i}^{-1} \tilde{\omega}_{i}^{-1} \tilde{e}_{i} \quad \text { for } a_{i} \in \mathbb{K}^{*} .
\end{aligned}
$$

Proof. Suppose $\varphi: \mathfrak{u}_{r, s} \rightarrow \mathfrak{u}_{r^{\prime}, s^{\prime}}$ is a Hopf algebra isomorphism. Write the generators of $\mathfrak{u}_{r^{\prime}, s^{\prime}}$ as $\tilde{e}_{i}, \tilde{f}_{i}, \tilde{\omega}_{i}$ and $\tilde{\omega}_{i}^{\prime}$. Because

$$
\Delta\left(\varphi\left(e_{i}\right)\right)=(\varphi \otimes \varphi)\left(\Delta\left(e_{i}\right)\right)=\varphi\left(e_{i}\right) \otimes 1+\varphi\left(\omega_{i}\right) \otimes \varphi\left(e_{i}\right),
$$

we have $\varphi\left(e_{i}\right) \in P_{1, \varphi\left(\omega_{i}\right)}\left(\mathfrak{u}_{r^{\prime}, s^{\prime}}\right)$ for $i=1,2$. As $\varphi$ is an isomorphism, $\varphi\left(\omega_{i}\right) \in \mathbb{K} \tilde{G}$. Lemma 4.3(1) implies that $\varphi\left(\omega_{i}\right) \in\left\{\tilde{\omega}_{j}, \tilde{\omega}_{j}^{\prime-1} \mid j=1,2\right\}$. Let $\pi:\{1,2\} \rightarrow\{1,2\}$ be a permutation. We distinguish four cases:

(i) There is some $\pi$ such that $\varphi\left(\omega_{i}\right)=\tilde{\omega}_{\pi(i)}$ and $\varphi\left(e_{i}\right)=\alpha\left(1-\tilde{\omega}_{\pi(i)}\right)+\beta \tilde{e}_{\pi(i)}$ for $i=1,2$ and $\alpha, \beta \in \mathbb{K}$. 
(ii) There is some $\pi$ such that $\varphi\left(\omega_{i}\right)=\tilde{\omega}_{\pi(i)}^{\prime-1}$ and $\varphi\left(e_{i}\right)=\alpha^{\prime}\left(1-\tilde{\omega}_{\pi(i)}^{\prime-1}\right)+\beta^{\prime} \tilde{f}_{\pi(i)} \tilde{\omega}_{\pi(i)}^{\prime-1}$ for $i=1,2$ and $\alpha^{\prime}, \beta^{\prime} \in \mathbb{K}$.

(iii) $\varphi\left(\omega_{1}\right)=\tilde{\omega}_{\pi(1)}$, but $\varphi\left(\omega_{2}\right)=\tilde{\omega}_{\pi(2)}^{\prime-1}$.

(iv) $\varphi\left(\omega_{1}\right)=\tilde{\omega}_{\pi(1)}^{\prime-1}$, but $\varphi\left(\omega_{2}\right)=\tilde{\omega}_{\pi(2)}$.

Case (i): In this case, we claim $\varphi\left(\omega_{i}\right)=\tilde{\omega}_{i}$. Assume otherwise, that is, $\varphi\left(\omega_{1}\right)=\tilde{\omega}_{2}$ and $\varphi\left(\omega_{2}\right)=\tilde{\omega}_{1}$. Applying $\varphi$ to the relation $\omega_{1} e_{1}=r s^{-1} e_{1} \omega_{1}$ yields

$$
\begin{aligned}
\varphi\left(\omega_{1}\right) \varphi\left(e_{1}\right) & =r s^{-1} \varphi\left(e_{1}\right) \varphi\left(\omega_{1}\right), \text { whence } \\
\tilde{\omega}_{2}\left(\alpha\left(1-\tilde{\omega}_{2}\right)+\beta \tilde{e}_{2}\right) & =r s^{-1}\left(\alpha\left(1-\tilde{\omega}_{2}\right)+\beta \tilde{e}_{2}\right) \tilde{\omega}_{2} .
\end{aligned}
$$

Since $r \neq s$, this forces $\alpha$ to be 0 , so that $\varphi\left(e_{1}\right)=\beta \tilde{e}_{2}$ for some $\beta \neq 0$. Also, because $\tilde{\omega}_{2} \tilde{e}_{2}=r_{2}^{\prime} s_{2}^{\prime-1} \tilde{e}_{2} \tilde{\omega}_{2}$, it follows that $r^{\prime 3} s^{\prime-3}=r s^{-1}$.

Applying $\varphi$ to the relation $\omega_{1} e_{2}=s^{3} e_{2} \omega_{1}$, we get $\varphi\left(\omega_{1}\right) \varphi\left(e_{2}\right)=s^{3} \varphi\left(e_{2}\right) \varphi\left(\omega_{1}\right)$, that is, $\tilde{\omega}_{2} \tilde{e}_{1}=s^{3} \tilde{e}_{1} \tilde{\omega}_{1}$. But $\tilde{\omega}_{2} \tilde{e}_{1}=r^{\prime-3} \tilde{e}_{1} \tilde{\omega}_{1}$ in $\mathfrak{u}_{r^{\prime}, s^{\prime}}$. So $r^{\prime 3}=s^{-3}$. Similarly, the relation $\omega_{2} e_{1}=r^{-3} e_{1} \omega_{2}$ gives $s^{\prime-3}=r^{3}$. It then follows from $r^{\prime 3} s^{\prime-3}=r s^{-1}$ that $r^{2}=s^{2}$, a contradiction. So we proved $\varphi\left(\omega_{i}\right)=\tilde{\omega}_{i}$. Similarly, we have $\varphi\left(\omega_{i}^{\prime}\right)=\tilde{\omega}_{i}^{\prime}$.

Furthermore, applying $\varphi$ to the relations $\omega_{i} e_{j}=\left\langle\omega_{j}^{\prime}, \omega_{i}\right\rangle e_{j} \omega_{i}$ for $i, j \in\{1,2\}$, we get $\left\langle\omega_{j}^{\prime}, \omega_{i}\right\rangle=\left\langle\tilde{\omega}_{j}^{\prime}, \tilde{\omega}_{i}\right\rangle$ for $i, j \in\{1,2\}$, that is,

$$
r^{\prime 3} s^{-3}=r^{3} s^{-3}, \quad r^{\prime} s^{\prime-1}=r s^{-1}, \quad r^{-3}=r^{-3}, \quad s^{\prime 3}=s^{3} .
$$

Hence, $\left(r^{\prime}, s^{\prime}\right)=\zeta(r, s)$, where $\zeta$ is a 3rd root of unity. As $\varphi$ preserves relation (G4), $\varphi\left(e_{i}\right)=a_{i} \tilde{e}_{i}$ and $\varphi\left(f_{i}\right)=\zeta^{\delta_{i, 1}} a_{i}^{-1} \tilde{f}_{i}$ for $a_{i} \in \mathbb{K}^{*}$, so $\varphi$ is a diagonal isomorphism.

Case (ii): In this case

$$
\varphi\left(\omega_{i}\right)=\tilde{\omega}_{\pi(i)}^{\prime-1} \text { for some } \pi(i) \quad \text { and } \quad \varphi\left(e_{i}\right)=\alpha^{\prime}\left(1-\tilde{\omega}_{\pi(i)}^{\prime-1}\right)+\beta^{\prime} \tilde{f}_{\pi(i)} \tilde{\omega}_{\pi(i)}^{\prime-1} .
$$

In fact, applying $\varphi$ to the relation $\omega_{i} e_{i}=r_{i} s_{i}^{-1} e_{i} \omega_{i}$ gives $\alpha^{\prime}=0$, so we have $\varphi\left(e_{i}\right)=\beta^{\prime} \tilde{f}_{\pi(i)} \tilde{\omega}_{\pi(i)}^{\prime-1}$. We claim that $\varphi\left(\omega_{i}\right)=\tilde{\omega}_{i}^{\prime-1}$. Assume otherwise, that is, $\varphi\left(\omega_{1}\right)=\tilde{\omega}_{2}^{\prime-1}$ and $\varphi\left(\omega_{2}\right)=\tilde{\omega}_{1}^{\prime-1}$. Then $\varphi\left(e_{1}\right)=a_{1} \tilde{f}_{2} \tilde{\omega}_{2}^{\prime-1}$ and $\varphi\left(e_{2}\right)=a_{2} \tilde{f}_{1} \tilde{\omega}_{1}^{\prime-1}$. Apply $\varphi$ to the relation $\omega_{1} e_{1}=r s^{-1} e_{1} \omega_{1}$ to get $r s^{-1}=\left(r^{\prime 3} s^{\prime-3}\right)^{-1}$, to the relation $\omega_{2} e_{1}=r^{-3} e_{1} \omega_{2}$ to get $r^{-3}=r^{\prime 3}$, and to the relation $\omega_{1} e_{2}=s^{3} e_{2} \omega_{1}$ to get $s^{3}=s^{\prime-3}$. So we have $\left(r s^{-1}\right)^{2}=1$, a contradiction. Hence, $\varphi\left(\omega_{i}\right)=\tilde{\omega}_{i}^{\prime-1}, \varphi\left(\omega_{i}^{\prime}\right)=\tilde{\omega}_{i}^{-1}$ and $\varphi\left(e_{i}\right)=a_{i} \tilde{f}_{i} \tilde{\omega}_{i}^{\prime-1}$, where $a_{i} \in \mathbb{K}^{*}$.

Apply $\varphi$ to the relation $\omega_{1} e_{1}=r s^{-1} e_{1} \omega_{1}$ to get $r s^{-1}=\left(r^{\prime} s^{\prime-1}\right)^{-1}$, to the relation $\omega_{1} e_{2}=s^{3} e_{2} \omega_{1}$ to get $s^{3}=r^{\prime 3}$ and to the relation $\omega_{2} e_{1}=r^{-3} e_{1} \omega_{2}$ to get $r^{-3}=s^{-3}$. So we have $\left(r^{\prime}, s^{\prime}\right)=\zeta(s, r)$, where $\zeta$ is a 3rd root of unity. In order that $\varphi$ preserve the relation (G4), one has to take $\varphi\left(f_{i}\right)=\zeta^{\delta_{i, 1}} a_{i}^{-1} \tilde{\omega}_{i}^{-1} \tilde{e}_{i}$, where $a_{i} \in \mathbb{K}^{*}$. Also, we can easily check that this $\varphi$ does preserve the Serre relations (G5) and (G6).

Case (iii): We claim that this case is impossible. First, we assume that $\varphi\left(\omega_{1}\right)=\tilde{\omega}_{1}$ and $\varphi\left(\omega_{2}\right)=\tilde{\omega}_{2}^{\prime-1}$. Then we have $\varphi\left(e_{1}\right)=a_{1} \tilde{e}_{1}$ and $\varphi\left(e_{2}\right)=a_{2} \tilde{f}_{2} \tilde{\omega}_{2}^{\prime-1}$, where 
$a_{1}, a_{2} \in \mathbb{K}^{*}$. Apply $\varphi$ to the relation $\omega_{1} e_{2}=s^{3} e_{2} \omega_{1}$ to get $s^{3}=s^{\prime-3}$ and to the relation $\omega_{2} e_{1}=r^{-3} e_{1} \omega_{2}$ to get $r^{-3}=s^{\prime 3}$. So we get $r^{3}=s^{3}$, a contradiction.

Next, we assume that $\varphi\left(\omega_{1}\right)=\tilde{\omega}_{2}$ and $\varphi\left(\omega_{2}\right)=\tilde{\omega}_{1}^{\prime-1}$. Then we have $\varphi\left(e_{1}\right)=a_{1} \tilde{e}_{2}$ and $\varphi\left(e_{2}\right)=a_{2} \tilde{f}_{1} \tilde{\omega}_{1}^{\prime-1}$, where $a_{1}, a_{2} \in \mathbb{K}^{*}$. Apply $\varphi$ to the relation $\omega_{1} e_{2}=s^{3} e_{2} \omega_{1}$ to get $s^{3}=r^{\prime 3}$ and to the relation $\omega_{2} e_{1}=r^{-3} e_{1} \omega_{2}$ to get $r^{-3}=r^{\prime-3}$. So we have $r^{3}=s^{3}$, a contradiction.

Case (iv): This case is similar to case (iii), and also impossible.

\section{5. $\mathfrak{u}_{r, s}\left(G_{2}\right)$ is a Drinfel'd double}

We will show that $\mathfrak{u} \cong D(\mathfrak{b})$ under a few assumptions. Let $\theta$ be a primitive $\ell$-th root of unity in $\mathbb{K}$, and write $r=\theta^{y}$ and $s=\theta^{z}$.

Lemma 5.1. Assume that $\left(3\left(y^{2}+z^{2}+y z\right), \ell\right)=1$ and $r^{-1}$ is a primitive $\ell$-th root of unity. Then $\left(\mathfrak{b}^{\prime}\right)^{\text {coop }} \cong \mathfrak{b}^{*}$ as Hopf algebras.

Proof. Define $\gamma_{j}, \eta_{j}$ for $j=1,2$ in $\mathfrak{b}^{*}$ as follows: The $\gamma_{j}$ are algebra homomorphisms with $\gamma_{j}\left(\omega_{i}\right)=\left\langle\omega_{j}^{\prime}, \omega_{i}\right\rangle$ and $\gamma_{j}\left(e_{i}\right)=0$ for $i=1$, 2. So they are grouplike elements in $\mathfrak{b}^{*}$. Let $\eta_{j}=\sum_{g \in G(\mathfrak{b})}\left(e_{j} g\right)^{*}$, where $G(\mathfrak{b})$ is the group generated by $\omega_{1}, \omega_{2}$ and the asterisk denotes the dual basis element relative to the PBW-basis of $\mathfrak{b}$. The isomorphism $\phi:\left(\mathfrak{b}^{\prime}\right)^{\operatorname{coop}} \rightarrow \mathfrak{b}^{*}$ is then defined by

$$
\phi\left(\omega_{j}^{\prime}\right)=\gamma_{j} \quad \text { and } \quad \phi\left(f_{j}\right)=\eta_{j} .
$$

We have to check that $\phi$ is a Hopf algebra homomorphism and a bijection.

First, we observe that the $\gamma_{j}$ are invertible elements of $\mathfrak{b}^{*}$ that commute with one another and that $\gamma_{j}^{\ell}=1$. Note that $\eta_{j}^{\ell}=0$, as it is 0 on any basis element of $\mathfrak{b}$. We calculate $\gamma_{j} \eta_{i} \gamma_{j}^{-1}$ : It is nonzero only on basis elements of the form $e_{i} \omega_{1}^{k_{1}} \omega_{2}^{k_{2}}$, and on any such element it takes the value

$\left(\gamma_{j} \otimes \eta_{i} \otimes \gamma_{j}^{-1}\right)\left(\left(e_{i} \otimes 1 \otimes 1+\omega_{i} \otimes e_{i} \otimes 1+\omega_{i} \otimes \omega_{i} \otimes e_{i}\right)\left(\omega_{1}^{k_{1}} \omega_{2}^{k_{2}}\right)^{\otimes 3}\right)$

$$
=\gamma_{j}\left(\omega_{i} \omega_{1}^{k_{1}} \omega_{2}^{k_{2}}\right) \eta_{i}\left(e_{i} \omega_{1}^{k_{1}} \omega_{2}^{k_{2}}\right) \gamma_{j}^{-1}\left(\omega_{1}^{k_{1}} \omega_{2}^{k_{2}}\right)=\gamma_{j}\left(\omega_{i}\right)=\left\langle\omega_{j}^{\prime}, \omega_{i}\right\rangle
$$

Thus we have $\gamma_{j} \eta_{i} \gamma_{j}^{-1}=\left\langle\omega_{j}^{\prime}, \omega_{i}\right\rangle \eta_{i}$, which corresponds to relation (G3) for $\mathfrak{b}^{\prime}$. Next, we check relation (G6): We compute

$$
\begin{aligned}
\left(\eta_{2}^{2} \eta_{1}\right)\left(e_{2}^{2} e_{1}\right)= & \left(\eta_{2} \otimes \eta_{2} \otimes \eta_{1}\right)\left(\left(e_{2} \otimes 1 \otimes 1+\omega_{2} \otimes e_{2} \otimes 1+\omega_{2} \otimes \omega_{2} \otimes e_{2}\right)^{2}\right. \\
& \left.\times\left(e_{1} \otimes 1 \otimes 1+\omega_{1} \otimes e_{1} \otimes 1+\omega_{1} \otimes \omega_{1} \otimes e_{1}\right)\right) \\
= & \left(\eta_{2} \otimes \eta_{2} \otimes \eta_{1}\right)\left(e_{2} \omega_{2} \omega_{1} \otimes e_{2} \omega_{1} \otimes e_{1}+\omega_{2} e_{2} \omega_{1} \otimes e_{2} \omega_{1} \otimes e_{1}\right) \\
= & \left(\eta_{2} \otimes \eta_{2} \otimes \eta_{1}\right)\left(\left(1+r^{3} s^{-3}\right) e_{2} \omega_{2} \omega_{1} \otimes e_{2} \omega_{1} \otimes e_{1}\right)=1+r^{3} s^{-3},
\end{aligned}
$$

and similarly $\left(\eta_{2}^{2} \eta_{1}\right)\left(e_{2} E_{12}\right)=0$. Thus, for any $k_{1}, k_{2}$, we have

$$
\left(\eta_{2}^{2} \eta_{1}\right)\left(e_{2}^{2} e_{1} \omega_{1}^{k_{1}} \omega_{2}^{k_{2}}\right)=1+r^{3} s^{-3} \quad \text { and } \quad\left(\eta_{2}^{2} \eta_{1}\right)\left(e_{2} E_{12} \omega_{1}^{k_{1}} \omega_{2}^{k_{2}}\right)=0 .
$$


On all other basis elements, $\eta_{2}^{2} \eta_{1}$ is 0 . Therefore, we have

$$
\eta_{2}^{2} \eta_{1}=\sum_{g \in G}\left(1+r^{3} s^{-3}\right)\left(e_{2}^{2} e_{1} g\right)^{*}
$$

Similarly, we calculate

$$
\begin{aligned}
& \left(\eta_{2} \eta_{1} \eta_{2}\right)\left(e_{2}^{2} e_{1}\right) \\
& \quad=\left(\eta_{2} \otimes \eta_{1} \otimes \eta_{2}\right)\left(e_{2} \omega_{2} \omega_{1} \otimes \omega_{2} e_{1} \otimes e_{2}+\omega_{2} e_{2} \omega_{1} \otimes \omega_{2} e_{1} \otimes e_{2}\right) \\
& \quad=r^{-3}+s^{-3} \\
& \left(\eta_{2} \eta_{1} \eta_{2}\right)\left(e_{2} E_{12}\right) \\
& \quad=\left(\eta_{2} \eta_{1} \eta_{2}\right)\left(e_{2} e_{1} e_{2}-s^{3} e_{2}^{2} e_{1}\right) \\
& \quad=\left(\eta_{2} \otimes \eta_{1} \otimes \eta_{2}\right)\left(\omega_{2} \omega_{1} e_{2} \otimes \omega_{2} e_{1} \otimes e_{2}+e_{2} \omega_{1} \omega_{2} \otimes e_{1} \omega_{2} \otimes e_{2}\right)-1-r^{-3} s^{3} \\
& \quad=1-r^{-3} s^{3} .
\end{aligned}
$$

So we have

$$
\eta_{2} \eta_{1} \eta_{2}=\sum_{g \in G}\left(\left(r^{-3}+s^{-3}\right)\left(e_{2}^{2} e_{1} g\right)^{*}+\left(1-r^{-3} s^{3}\right)\left(e_{2} E_{12} g\right)^{*}\right) .
$$

We compute

$$
\left(\eta_{1} \eta_{2}^{2}\right)\left(e_{2}^{2} e_{1}\right)=r^{-3}\left(r^{-3}+s^{-3}\right) \quad \text { and } \quad\left(\eta_{1} \eta_{2}^{2}\right)\left(e_{2} E_{12}\right)=s^{3}\left(s^{-6}-r^{-6}\right) .
$$

So we have

$$
\eta_{1} \eta_{2}^{2}=\sum_{g \in G}\left(r^{-3}\left(r^{-3}+s^{-3}\right)\left(e_{2}^{2} e_{1} g\right)^{*}+s^{3}\left(s^{-6}-r^{-6}\right)\left(e_{2} E_{12} g\right)^{*}\right) .
$$

We use (5-1)-(5-3) to establish the relation

$$
r^{3} s^{3} \eta_{1} \eta_{2}^{2}-\left(r^{3}+s^{3}\right) \eta_{2} \eta_{1} \eta_{2}+\eta_{2}^{2} \eta_{1}=0 .
$$

Similarly, it is easy to verify that

$$
\begin{aligned}
\eta_{2} \eta_{1}^{4}-(r+s)\left(r^{2}+s^{2}\right) \eta_{1} \eta_{2} \eta_{1}^{3}+ & r s\left(r^{2}+s^{2}\right)\left(r^{2}+r s+s^{2}\right) \eta_{1}^{2} \eta_{2} \eta_{1}^{2} \\
& -(r s)^{3}(r+s)\left(r^{2}+s^{2}\right) \eta_{1}^{3} \eta_{2} \eta_{1}+(r s)^{6} \eta_{1}^{4} \eta_{2}=0 .
\end{aligned}
$$

Therefore, $\phi$ is an algebra homomorphism.

Now we will check that $\phi$ preserves coproducts. We have already seen that $\gamma_{i}$ is a group-like element in $\mathfrak{b}^{*}$. We calculate

$$
\begin{aligned}
& \Delta\left(\eta_{i}\right)\left(e_{i} \omega_{1}^{j_{1}} \omega_{2}^{j_{2}} \otimes \omega_{1}^{k_{1}} \omega_{2}^{k_{2}}\right)=\eta_{i}\left(e_{i} \omega_{1}^{j_{1}+k_{1}} \omega_{2}^{j_{2}+k_{2}}\right)=1, \\
& \Delta\left(\eta_{i}\right)\left(\omega_{1}^{j_{1}} \omega_{2}^{j_{2}} \otimes e_{i} \omega_{1}^{k_{1}} \omega_{2}^{k_{2}}\right)=\eta_{i}\left(\omega_{1}^{j_{1}} \omega_{2}^{j_{2}} e_{i} \omega_{1}^{k_{1}} \omega_{2}^{k_{2}}\right)=\left\langle\omega_{i}^{\prime}, \omega_{1}\right\rangle^{j_{1}}\left\langle\omega_{i}^{\prime}, \omega_{2}\right\rangle^{j_{2}} .
\end{aligned}
$$


These are the only basis elements of $\mathfrak{b} \otimes \mathfrak{b}$ on which $\Delta\left(\eta_{i}\right)$ is nonzero. As a consequence, we have

$$
\begin{aligned}
& \left(\eta_{i} \otimes 1+\gamma_{i} \otimes \eta_{i}\right)\left(e_{i} \omega_{1}^{j_{1}} \omega_{2}^{j_{2}} \otimes \omega_{1}^{k_{1}} \omega_{2}^{k_{2}}\right)=1, \\
& \left(\eta_{i} \otimes 1+\gamma_{i} \otimes \eta_{i}\right)\left(\omega_{1}^{j_{1}} \omega_{2}^{j_{2}} \otimes e_{i} \omega_{1}^{k_{1}} \omega_{2}^{k_{2}}\right)=\left\langle\omega_{i}^{\prime}, \omega_{1}\right\rangle^{j_{1}}\left\langle\omega_{i}^{\prime}, \omega_{2}\right\rangle^{j_{2}} .
\end{aligned}
$$

So $\Delta\left(\eta_{i}\right)=\eta_{i} \otimes 1+\gamma_{i} \otimes \eta_{i}$. This proves that $\phi$ is a Hopf algebra homomorphism.

Finally, we prove that $\phi$ is bijective. As $\mathfrak{b}^{*}$ and $\left(\mathfrak{b}^{\prime}\right)^{\text {coop }}$ have the same dimension, it suffices to show that $\phi$ is injective. By [Montgomery 1993], we need only show that $\phi$, when restricted to $\left(\mathfrak{b}^{\prime}\right)_{1}^{\text {coop }}$, is injective. Lemma 4.2 yields $\left(\mathfrak{b}^{\prime}\right)_{1}^{\text {coop }}=\mathbb{K} G\left(\mathfrak{b}^{\prime}\right)+\sum_{i=1}^{2} \mathbb{K} f_{i} G\left(\mathfrak{b}^{\prime}\right)$, where $G\left(\mathfrak{b}^{\prime}\right)$ is the group generated by $\omega_{1}^{\prime}$ and $\omega_{2}^{\prime}$. First, we claim that

$$
\operatorname{span}_{\mathbb{R}}\left\{\gamma_{1}^{k_{1}} \gamma_{2}^{k_{2}} \mid 0 \leq k_{i}<\ell\right\}=\operatorname{span}_{\mathbb{K}}\left\{\left(\omega_{1}^{k_{1}} \omega_{2}^{k_{2}}\right)^{*} \mid 0 \leq k_{i}<\ell\right\} .
$$

This is equivalent to the statement that the $\gamma_{1}^{k_{1}} \gamma_{2}^{k_{2}}$ span the space of characters over $\mathbb{K}$ of the finite group $\mathbb{Z} / \ell \mathbb{Z} \times \mathbb{Z} / \ell \mathbb{Z}$ generated by $\omega_{1}$ and $\omega_{2}$. We have assumed that $\mathbb{K}$ contains a primitive $\ell$-th root of unity. Therefore, the irreducible characters of this group are the functions $\chi_{i_{1}, i_{2}}$ given by $\chi_{i_{1}, i_{2}}\left(\omega_{1}^{k_{1}} \omega_{2}^{k_{2}}\right)=\theta^{i_{1} k_{1}+i_{2} k_{2}}$, where $\theta$ is a primitive $\ell$-th root of unity in $\mathbb{K}$. Note that $\gamma_{1}=\chi_{y-z,-3 y}$ and $\gamma_{2}=\chi_{3 z, 3(y-z)}$. We must show that, given $i_{1}, i_{2}$, there are $k_{1}, k_{2}$ such that $\chi_{i_{1}, i_{2}}=\gamma_{1}^{k_{1}} \gamma_{2}^{k_{2}}$, which is equivalent to the existence of a solution to the matrix equation $A K=I$ in $\mathbb{Z} / \ell \mathbb{Z}$ (as these are powers of $\theta$ ), where $A=\left(\begin{array}{cc}y-z & 3 z \\ -3 y & 3(y-z)\end{array}\right), K$ is the transpose of $\left(k_{1}, k_{2}\right)$ and $I$ is the transpose of $\left(i_{1}, i_{2}\right)$. The determinant of the coefficient matrix $A$ is $3\left(y^{2}+z^{2}+y z\right)$, which is invertible in $\mathbb{Z} / \ell \mathbb{Z}$ by the hypothesis of the lemma. Therefore (5-4) holds. In particular, this implies that the matrix

$$
\left(\left(\gamma_{1}^{k_{1}} \gamma_{2}^{k_{2}}\right)\left(\omega_{1}^{j_{1}} \omega_{2}^{j_{2}}\right)\right)_{\bar{k} \times \bar{j}}
$$

is invertible, and that $\phi$ is bijection on group-like elements.

Next we will show for each $i=1$ and $i=2$ the matrix

$$
\left(\left(\eta_{i} \gamma_{1}^{k_{1}} \gamma_{2}^{k_{2}}\right)\left(e_{i} \omega_{1}^{j_{1}} \omega_{2}^{j_{2}}\right)\right)_{\bar{k} \times \bar{j}}
$$

is invertible. This will complete the proof that $\phi$ is injective on $\left(\mathfrak{b}^{\prime}\right)_{1}^{\text {coop }}$, as desired. We will show that the matrix is block upper-triangular. Each matrix entry is

$$
\left(\eta_{i} \otimes \gamma_{1}^{k_{1}} \gamma_{2}^{k_{2}}\right)\left(\Delta\left(e_{i}\right) \Delta\left(\omega_{1}^{j_{1}} \omega_{2}^{j_{2}}\right)\right)=\left(\eta_{i} \otimes \gamma_{1}^{k_{1}} \gamma_{2}^{k_{2}}\right)\left(e_{i} \omega_{1}^{j_{1}} \omega_{2}^{j_{2}} \otimes \omega_{1}^{j_{1}} \omega_{2}^{j_{2}}\right) .
$$

Thus, (5-6) is precisely the invertible matrix (5-5).

Recall that the Drinfel'd double $D(\mathfrak{b})$ of the finite-dimensional Hopf algebra $\mathfrak{b}$ is a Hopf algebra whose underlying coalgebra is $\mathfrak{b} \otimes\left(\mathfrak{b}^{*}\right)^{\text {coop }}$ (that is, the vector space $\mathfrak{b} \otimes\left(\mathfrak{b}^{*}\right)^{\text {coop }}$ with the tensor product coalgebra structure). As an algebra, $D(\mathfrak{b})$ 
contains the subalgebras $\mathfrak{b} \otimes 1 \cong \mathfrak{b}$ and $1 \otimes \mathfrak{b}^{*} \cong \mathfrak{b}^{*}$, and if $a \in \mathfrak{b}$ and $b \in\left(\mathfrak{b}^{*}\right)^{\text {coop }}$, then $(a \otimes 1)(1 \otimes b)=a \otimes b$ and

$$
(1 \otimes b)(a \otimes 1)=\sum b_{(1)}\left(S^{-1} a_{(1)}\right) b_{(3)}\left(a_{(3)}\right) a_{(2)} \otimes b_{(2)},
$$

where $S^{-1}$ is the composition inverse of the antipode $S$ for $\mathfrak{b}$.

Theorem 5.2. Assume that $\left(3\left(y^{2}+z^{2}+y z\right), \ell\right)=1$. Then $D(\mathfrak{b}) \cong \mathfrak{u}_{r, s}\left(G_{2}\right)$ as Hopf algebras.

Proof. We denote the image $e_{i} \otimes 1$ of $e_{i}$ in $D(\mathfrak{b})$ by $\check{e}_{i}$, and similarly for $\omega_{i}, \eta_{i}$ and $\gamma_{i}$. Define $\psi: D(\mathfrak{b}) \rightarrow \mathfrak{u}_{r, s}\left(G_{2}\right)$ on the generators by

$$
\begin{aligned}
\psi\left(\check{e}_{i}\right) & =e_{i}, & \psi\left(\check{\eta}_{i}\right) & =\left(s_{i}-r_{i}\right) f_{i}, \\
\psi\left(\check{\omega}_{i}^{ \pm 1}\right) & =\omega_{i}^{ \pm 1}, & \psi\left(\check{\gamma}_{i}^{ \pm 1}\right) & =\left(\omega_{i}^{\prime}\right)^{ \pm 1} .
\end{aligned}
$$

Then the proof is the same as that of [Hu and Wang 2009, Theorem 6.2].

\section{Integrals and ribbon elements}

Integrals play a basic role in the structure theory of finite-dimensional Hopf algebras $H$ and their duals $H^{*}$. In this section, we compute the left and right integrals in the Borel subalgebra $\mathfrak{b}$ of $\mathfrak{u}_{r, s}\left(G_{2}\right)$ and the distinguished group-like elements of $\mathfrak{b}$ and $\mathfrak{b}^{*}$. We use this information to determine that $\mathfrak{u}_{r, s}\left(G_{2}\right)$ has a ribbon element when $\mathfrak{u}_{r, s}\left(G_{2}\right) \cong D(\mathfrak{b})$.

Let $H$ be a finite-dimensional Hopf algebra. An element $y \in H$ is a left integral if $a y=\varepsilon(a) y$ for all $a \in H$, and a right integral if $y a=\varepsilon(a) y$. The left and right integrals form a one-dimensional ideals $\int_{H}^{l}$ and $\int_{H}^{r}$, respectively, of $H$, and $S_{H}\left(\int_{H}^{r}\right)=\int_{H}^{l}$ under the antipode $S_{H}$ of $H$.

When $y \neq 0$ is a left integral of $H$, there exists a unique group-like element $\gamma$ in the dual algebra $H^{*}$ (the so-called distinguished group-like element of $H^{*}$ ) such that $y a=\gamma(a) y$. If we had begun instead with a right integral $y^{\prime} \in H$, then we would have $a y^{\prime}=\gamma^{-1}(a) y^{\prime}$. This is an easy consequence of the fact that group-like elements are invertible, and can be found in [Montgomery 1993, page 22].

Now if $\lambda \neq 0$ is right integral of $H^{*}$, then there exists a unique group-like element $g$ of $H$ (the distinguished group-like element of $H$ ) such that $\xi \lambda=\xi(g) \lambda$ for all $\xi \in H^{*}$. The algebra $H$ is unimodular (that is, $\int_{H}^{l}=\int_{H}^{r}$ ) if and only if $\gamma=\varepsilon$; and the dual algebra $H^{*}$ is unimodular if and only if $g=1$.

The left and right $H^{*}$-module actions on $H$ are given by

$$
\xi \rightarrow a=\sum a_{(1)} \xi\left(a_{(2)}\right) \quad \text { and } \quad a<\xi=\sum \xi\left(a_{(1)}\right) a_{(2)}
$$

for all $\xi \in H^{*}$ and $a \in H$. In particular, $\varepsilon \rightarrow a=a=a \leftarrow \varepsilon$ for all $a \in H$. In [1976], Radford found a remarkable expression relating the antipode, the distinguished 
group-like elements $\gamma$ and $g$, and the $H^{*}$-action, namely,

$$
S^{4}(a)=g\left(\gamma \rightarrow a<\gamma^{-1}\right) g^{-1} \text { for all } a \in H .
$$

This formula was crucial in [Kauffman and Radford 1993] for determining a necessary and sufficient condition for a Drinfel'd double of a Hopf algebra to have a ribbon element.

A finite-dimensional Hopf algebra $H$ is quasitriangular if there is an invertible element $R=\sum x_{i} \otimes y_{i}$ in $H \otimes H$ such that $\Delta^{\mathrm{op}}(a) R=R \Delta(a)$ for all $a \in H$, and $R$ satisfies the relations $(\Delta \otimes \mathrm{id}) R=R_{1,3} R_{2,3}$ and (id $\otimes \Delta$ ) $R=R_{1,3} R_{1,2}$, where $R_{1,2}=\sum x_{i} \otimes y_{i} \otimes 1, R_{1,3}=\sum x_{i} \otimes 1 \otimes y_{i}$, and $R_{2,3}=\sum 1 \otimes x_{i} \otimes y_{i}$. Suppose $u=\sum S\left(y_{i}\right) x_{i}$. Then $c=u S(u)$ is central in $H$ and is referred to as the Casimir.

An element $v \in H$ is a quasiribbon element of a quasitriangular Hopf algebra $(H, R)$ if

$$
v^{2}=c, \quad S(v)=v, \quad \varepsilon(v)=1, \quad \Delta(v)=\left(R_{2,1} R_{1,2}\right)^{-1}(v \otimes v),
$$

where $R_{2,1}=\sum y_{i} \otimes x_{i}$ and $R_{1,2}=R$. If $v$ is also central in $H$, then $v$ is a ribbon element, and $(H, R, v)$ is called a ribbon Hopf algebra. Ribbon elements provide an effective means of constructing invariants of knots and links; see [Kauffman and Radford 1993; Reshetikhin and Turaev 1990; 1991; Reshetikhin et al. 1989]. The Drinfel'd double $D(A)$ of a finite-dimensional Hopf algebra $A$ is quasitriangular, and Kauffman and Radford [1993] have provided a simple criterion for $D(A)$ to have a ribbon element.

Theorem 6.1. Assume that $A$ is a finite-dimensional Hopf algebra, and let $g$ and $\gamma$ be the distinguished group-like elements of $A$ and $A^{*}$, respectively. Then

(i) $(D(A), R)$ has a quasiribbon element if and only if there exist group-like elements $h \in A$ and $\delta \in A^{*}$ such that $h^{2}=g$ and $\delta^{2}=\gamma$.

(ii) $(D(A), R)$ has a ribbon element if and only if there exist $h$ and $\delta$ as in (i) such that

$$
S^{2}(a)=h\left(\delta \rightarrow a \leftarrow \delta^{-1}\right) h^{-1} \quad \text { for all } a \in A .
$$

Next we compute a left integral and a right integral in $\mathfrak{b}$.

Proposition 6.2. Let

$$
t=\prod_{i=1}^{2}\left(1+\omega_{i}+\cdots+\omega_{i}^{\ell-1}\right) \quad \text { and } \quad x=E_{2}^{\ell-1} E_{12}^{\ell-1} E_{11212}^{\ell-1} E_{112}^{\ell-1} E_{1112}^{\ell-1} E_{1}^{\ell-1} .
$$

Then $y=t x$ and $y^{\prime}=x t$ are respectively a left integral and a right integral in $\mathfrak{b}$.

Proof. To prove $y=t x$ is a left integral in $\mathfrak{b}$, we need to show that $b y=\varepsilon(b) y$ for all $b \in \mathfrak{b}$. It suffices to show this for the generators $\omega_{k}$ and $e_{k}$ for $k=1,2$, as the counit $\varepsilon$ is an algebra homomorphism. 
Observe that $\omega_{k} t=t=\varepsilon\left(\omega_{k}\right) t$ for all $k=1,2$, because the $\omega_{i}$ commute and $\omega_{k}\left(1+\omega_{k}+\cdots+\omega_{k}^{\ell-1}\right)=1+\omega_{k}+\cdots+\omega_{k}^{\ell-1}$. From that, the relation $\omega_{k} y=\varepsilon\left(\omega_{k}\right) y$ is clear for all $k$.

Next we compute $e_{k} y$. By a simple calculation, we get

$$
e_{k} t=\prod_{i=1}^{2}\left(1+\left\langle\omega_{k}^{\prime}, \omega_{i}\right\rangle^{-1} \omega_{i}+\cdots+\left\langle\omega_{k}^{\prime}, \omega_{i}\right\rangle^{-(\ell-1)} \omega_{i}^{\ell-1}\right) e_{k} .
$$

So it suffices to check that $e_{k} x=0=\varepsilon\left(e_{k}\right) x$.

Note that $e_{2} x=0$. We want to show that $e_{1}$ can be moved across the terms $E_{2}^{\ell-1} E_{12}^{\ell-1} E_{11212}^{\ell-1} E_{112}^{\ell-1} E_{1112}^{\ell-1}$ preceding $E_{1}^{\ell-1}$. We have

$$
\begin{aligned}
e_{1} E_{2}^{\ell-1} E_{12}^{\ell-1} E_{11212}^{\ell-1} E_{112}^{\ell-1} E_{1112}^{\ell-1} & =s^{3(\ell-1)} E_{2}^{\ell-1} e_{1} E_{12}^{\ell-1} E_{11212}^{\ell-1} E_{112}^{\ell-1} E_{1112}^{\ell-1} \\
& =r^{\ell-1} s^{5(\ell-1)} E_{2}^{\ell-1} E_{12}^{\ell-1} e_{1} E_{11212}^{\ell-1} E_{112}^{\ell-1} E_{1112}^{\ell-1} \\
& =r^{4(\ell-1)} s^{8(\ell-1)} E_{2}^{\ell-1} E_{12}^{\ell-1} E_{11212}^{\ell-1} e_{1} E_{112}^{\ell-1} E_{1112}^{\ell-1} \\
& =r^{6(\ell-1)} s^{9(\ell-1)} E_{2}^{\ell-1} E_{12}^{\ell-1} E_{11212}^{\ell-1} E_{112}^{\ell-1} e_{1} E_{1112}^{\ell-1} \\
& =r^{9(\ell-1)} s^{9(\ell-1)} E_{2}^{\ell-1} E_{12}^{\ell-1} E_{11212}^{\ell-1} E_{112}^{\ell-1} E_{1112}^{\ell-1} e_{1},
\end{aligned}
$$

where the first equality follows from Lemma 3.4(6), the second from Lemmas 3.5(3) and 3.1(9), the third from Lemma 3.4(4), the fourth from Lemma 3.4(3) and the last from Lemma 3.4(2). Thus, we have $e_{1} x=0$, which implies the desired conclusion that $y$ is a left integral in $\mathfrak{b}$.

To prove that $y^{\prime}=x t$ is a right integral in $\mathfrak{b}$, it suffices to show that $x e_{j}=0$. Note that $x e_{1}=0$. By a similar argument and Lemma 3.5(2), (4), (1), Lemma 3.4(5), (1) and Lemma 3.1(4), (6), (8), (9), together with (2-5) and (2-6), we can move $e_{2}$ to the left until it is next to $E_{2}^{\ell-1}$, which gives zero.

A finite-dimensional Hopf algebra $H$ is semisimple if and only if $\varepsilon\left(\int_{H}^{l}\right) \neq 0$ or equivalently $\varepsilon\left(\int_{H}^{r}\right) \neq 0$. For the algebra $\mathfrak{b}, \quad y$ gives a basis for $\int_{\mathfrak{b}}^{l}$ and $y^{\prime}$ gives a basis for $\int_{\mathfrak{b}}^{r}$. Since $\varepsilon(y)=0=\varepsilon\left(y^{\prime}\right)$, we have this:

Proposition 6.3. The Hopf algebra $\mathfrak{b}$ is not semisimple.

We will compute the distinguished group-like elements of $\mathfrak{b}$ and $\mathfrak{b}^{*}$. The grouplike elements of $\mathfrak{b}^{*}$ are exactly the algebra homomorphisms $\operatorname{Alg}_{\mathbb{K}}(\mathfrak{b}, \mathbb{K})$, so to verify that a particular homomorphism is the distinguished group-like element, it suffices to compute its values on the generators.

Proposition 6.4. Write $2 \rho=10 \alpha_{1}+6 \alpha_{2}$, where $\rho$ is the half sum of positive roots. Let $\gamma \in \operatorname{Alg}_{\mathbb{K}}(\mathfrak{b}, \mathbb{K})$ be defined by

$$
\gamma\left(e_{k}\right)=0 \quad \text { and } \quad \gamma\left(\omega_{k}\right)=\left\langle\omega_{1}^{\prime}, \omega_{k}\right\rangle^{10}\left\langle\omega_{2}^{\prime}, \omega_{k}\right\rangle^{6} .
$$

Then $\gamma$ is the distinguished group-like element of $\mathfrak{b}^{*}$. 
Proof. It suffices to argue that $\gamma$ as in (6-1) satisfies $y a=\gamma(a) y$ for $a=e_{k}$ and $a=\omega_{k}$ for $k=1,2$ and for $y=t x$ given in Theorem 6.1. Recall from the proof of Proposition 6.2 that $x e_{k}=0$. Thus, $y e_{k}=t x e_{k}=0=\gamma\left(e_{k}\right) y$. We have for $k=1,2$

$$
\begin{aligned}
y \omega_{k}=t x \omega_{k} & =t\left(E_{2}^{\ell-1} E_{12}^{\ell-1} E_{11212}^{\ell-1} E_{112}^{\ell-1} E_{1112}^{\ell-1} E_{1}^{\ell-1}\right) \omega_{k} \\
& =\left\langle\omega_{1}^{\prime}, \omega_{k}\right\rangle^{-10(\ell-1)}\left\langle\omega_{2}^{\prime}, \omega_{k}\right\rangle^{-6(\ell-1)} t \omega_{k} x \\
& =\left\langle\omega_{1}^{\prime}, \omega_{k}\right\rangle^{10}\left\langle\omega_{2}^{\prime}, \omega_{k}\right\rangle^{6} t \omega_{k} x=\gamma\left(\omega_{k}\right) y .
\end{aligned}
$$

Under the assumptions of Lemma $5.1,\left(\mathfrak{b}^{\prime}\right)^{\text {coop }} \cong \mathfrak{b}^{*}$ as Hopf algebras, via the map $\phi:\left(\mathfrak{b}^{\prime}\right)^{\text {coop }} \rightarrow \mathfrak{b}^{*}$ under which $\phi\left(\omega_{j}^{\prime}\right)=\gamma_{j}$ and $\phi\left(f_{j}\right)=\eta_{j}$. (The $\gamma_{j}$ and $\eta_{j}$ are defined in the proof of Lemma 5.1). This allows us to define a Hopf pairing $\mathfrak{b}^{\prime} \times \mathfrak{b} \rightarrow \mathbb{K}$ whose values on generators are given by

$$
\left(f_{j} \mid e_{i}\right)=\delta_{i j} \quad \text { and } \quad\left(\omega_{j}^{\prime} \mid \omega_{i}\right)=\left\langle\omega_{j}^{\prime}, \omega_{i}\right\rangle,
$$

and are zero on all other pairs of generators. If we set $\omega_{2 \rho}^{\prime}:=\omega_{1}^{\prime 10} \omega_{2}^{\prime 6}$, then $\left(\omega_{2 \rho}^{\prime} \mid b\right)=\gamma(b)$ for all $b \in \mathfrak{b}$, that is, $\gamma=\left(\omega_{2 \rho}^{\prime} \mid \cdot\right)$.

Note that $\mathfrak{b}_{s^{-1}, r^{-1}} \cong\left(\mathfrak{b}^{\prime}\right)^{\text {coop }} \cong \mathfrak{b}^{*}$ as Hopf algebras. Under the isomorphism $\phi \psi^{-1}$ (where $\psi\left(f_{i}\right)=e_{i}$ and $\psi\left(\omega_{i}^{\prime}\right)=\omega_{i}$ ), a nonzero left integral of $\mathfrak{b}$ maps to a nonzero left integral of $\mathfrak{b}^{*}$, and likewise for right integrals. Thus, we have:

Proposition 6.5. Let $\lambda=v \eta$ and $\lambda^{\prime}=\eta v \in \mathfrak{b}^{*}$, where

$$
v=\prod_{i=1}^{2}\left(1+\gamma_{i}+\cdots+\gamma_{i}^{\ell-1}\right) \quad \text { and } \quad \eta=\eta_{2}^{\ell-1} \eta_{12}^{\ell-1} \eta_{11212}^{\ell-1} \eta_{112}^{\ell-1} \eta_{1112}^{\ell-1} \eta_{1}^{\ell-1},
$$

where

$$
\begin{aligned}
\eta_{12} & =\left[\eta_{1}, \eta_{2}\right]_{r^{3}}, & \eta_{112} & =\left[\eta_{1}, \eta_{12}\right]_{r^{2} s}, \\
\eta_{1112} & =\left[\eta_{1}, \eta_{112}\right]_{r s^{2}}, & \eta_{11212} & =\left[\eta_{112}, \eta_{12}\right]_{r s^{2}} .
\end{aligned}
$$

Then $\lambda$ is a left integral and $\lambda^{\prime}$ is a right integral of $\mathfrak{b}^{*}$.

Proposition 6.6. The element $g:=\omega_{2 \rho}^{-1}$ is the distinguished group-like element of $\mathfrak{b}$, and under the Hopf pairing in (6-2),

$$
\left(\omega_{i}^{\prime} \mid g\right)=\left\langle\omega_{i}^{\prime}, \omega_{1}\right\rangle^{-10}\left\langle\omega_{i}^{\prime}, \omega_{2}\right\rangle^{-6}=\gamma_{i}(g) \text { for } i=1,2 .
$$

Proof. Let $F=F_{2}^{\ell-1} F_{12}^{\ell-1} F_{11212}^{\ell-1} F_{112}^{\ell-1} F_{1112}^{\ell-1} F_{1}^{\ell-1}$. Then we have

$$
\omega_{k}^{\prime} F=\left\langle\omega_{k}^{\prime}, \omega_{1}\right\rangle^{-10}\left\langle\omega_{k}^{\prime}, \omega_{2}\right\rangle^{-6} F \omega_{k}^{\prime} .
$$

Since $\phi^{-1}\left(\lambda^{\prime}\right)=F\left(\prod_{i=1}^{2}\left(1+\omega_{i}^{\prime}+\cdots+\left(\omega_{i}^{\prime}\right)^{\ell-1}\right)\right)$, it follows that

$$
\gamma_{k} \lambda^{\prime}=\left\langle\omega_{k}^{\prime}, \omega_{1}\right\rangle^{-10}\left\langle\omega_{k}^{\prime}, \omega_{2}\right\rangle^{-6} \lambda^{\prime} \quad \text { and } \quad \eta_{k} \lambda^{\prime}=0
$$

Taking $g:=\omega_{2 \rho}^{-1}$, we have $\xi \lambda^{\prime}=\xi(g) \lambda^{\prime}$ for all $\xi \in \mathfrak{b}^{*}$. 
Theorem 6.7. Assume that $r$ and $s$ are $\ell$-th roots of unity. Then $D(\mathfrak{b})$ has a ribbon element.

Proof. By Proposition 6.6, $g=\omega_{2 \rho}^{-1}$ is the distinguished group-like element of $\mathfrak{b}$. There is a group-like element $h=\omega_{\rho}^{-1} \in \mathfrak{b}$ such that $h^{2}=g$. Because $\gamma=\left(\omega_{2 \rho}^{\prime} \mid \cdot\right)$ corresponds to $\omega_{2 \rho}^{\prime}$ under the isomorphism $\phi^{-1}: \mathfrak{b}^{*} \rightarrow\left(\mathfrak{b}^{\prime}\right)^{\text {coop }}$, there exists a $\delta=\left(\omega_{\rho}^{\prime} \mid \cdot\right) \in \mathfrak{b}^{*}$ such that $\delta^{2}=\gamma$, which is given by

$$
\delta\left(e_{k}\right)=0 \quad \text { and } \quad \delta\left(\omega_{k}\right)=\left\langle\omega_{1}^{\prime}, \omega_{k}\right\rangle^{5}\left\langle\omega_{2}^{\prime}, \omega_{k}\right\rangle^{3} \quad \text { for } k=1,2 .
$$

Then

$$
\begin{aligned}
h\left(\delta \rightarrow \omega_{k}-\delta^{-1}\right) h^{-1} & =\delta\left(\omega_{k}\right) \delta^{-1}\left(\omega_{k}\right) h \omega_{k} h^{-1}=\omega_{k}=S^{2}\left(\omega_{k}\right), \\
h\left(\delta \rightarrow e_{k} \leftarrow \delta^{-1}\right) h^{-1} & =\delta(1) \delta^{-1}\left(\omega_{k}\right) h e_{k} h^{-1} \\
& =\left\langle\omega_{1}^{\prime}, \omega_{k}\right\rangle^{-5}\left\langle\omega_{2}^{\prime}, \omega_{k}\right\rangle^{-3} h e_{k} h^{-1} \\
& =\left\langle\omega_{1}^{\prime}, \omega_{k}\right\rangle^{-5}\left\langle\omega_{2}^{\prime}, \omega_{k}\right\rangle^{-3}\left\langle\omega_{k}^{\prime}, \omega_{1}\right\rangle^{-5}\left\langle\omega_{k}^{\prime}, \omega_{2}\right\rangle^{-3} e_{k} \\
& =\omega_{k}^{-1} e_{k} \omega_{k}=S^{2}\left(e_{k}\right) .
\end{aligned}
$$

Then Theorem 6.1 implies the result.

Under the hypothesis of Theorem 5.2, $\mathfrak{u}_{r, s}\left(G_{2}\right) \cong D(\mathfrak{b})$, leading to this corollary:

Corollary 6.8. Assume that $r=\theta^{y}$ and $s=\theta^{z}$, where $\theta$ is a primitive $\ell$-th root of unity and $\left(3\left(y^{2}+z^{2}+y z\right), \ell\right)=1$. Then $\mathfrak{u}_{r, s}\left(G_{2}\right)$ has a ribbon element.

\section{References}

[Andruskiewitsch 2002] N. Andruskiewitsch, “About finite dimensional Hopf algebras", pp. 1-57 in Quantum symmetries in theoretical physics and mathematics (Bariloche, 2000), edited by R. Coquereaux et al., Contemp. Math. 294, Amer. Math. Soc., Providence, RI, 2002. MR 2003f:16059

[Andruskiewitsch and Schneider 1998] N. Andruskiewitsch and H.-J. Schneider, "Lifting of quantum linear spaces and pointed Hopf algebras of order $p^{3}$,, J. Algebra 209:2 (1998), 658-691. MR 99k:16075 Zbl 0919.16027

[Andruskiewitsch and Schneider 2002] N. Andruskiewitsch and H.-J. Schneider, "Pointed Hopf algebras", pp. 1-68 in New directions in Hopf algebras, edited by S. Montgomery and H.-J. Schneider, Math. Sci. Res. Inst. Publ. 43, Cambridge Univ. Press, Cambridge, 2002. MR 2003e:16043 Zbl 1011.16025

[Andruskiewitsch and Schneider 2006] N. Andruskiewitsch and H.-J. Schneider, "On the classification of finite-dimensional pointed Hopf algebras", preprint, 2006. To appear in Ann. Math. arXiv math.QA/0502157v3

[Bai and Hu 2008] X. Bai and N. Hu, "Two-parameter quantum groups of exceptional type $E$-series and convex PBW-type basis”, Algebra Colloq. 15:4 (2008), 619-636. MR 2451995 Zbl 05504520

[Beattie et al. 1999] M. Beattie, S. Dăscălescu, and L. Grünenfelder, "On the number of types of finite-dimensional Hopf algebras”, Inventiones Mathematicae 136:1 (1999), 1-7. MR 2000a:16068 Zbl 0922.16021 
[Beck 1994] J. Beck, "Convex bases of PBW type for quantum affine algebras", Comm. Math. Phys. 165:1 (1994), 193-199. MR 96b:17008 Zbl 0828.17016

[Benkart 1999] G. Benkart, "Down-up algebras and Witten's deformations of the universal enveloping algebra of $\mathfrak{s l}_{2}$ ”, pp. 29-45 in Recent progress in algebra (Taejon/Seoul, 1997), edited by S. G. Hahn et al., Contemp. Math. 224, Amer. Math. Soc., Providence, RI, 1999. MR 99m:17014 Zbl 0922.17007

[Benkart and Witherspoon 2004a] G. Benkart and S. Witherspoon, "Representations of two-parameter quantum groups and Schur-Weyl duality", pp. 65-92 in Hopf algebras, edited by J. Bergen et al., Lecture Notes in Pure and Appl. Math. 237, Dekker, New York, 2004. MR 2005g:17027 Zbl 1048.16021

[Benkart and Witherspoon 2004b] G. Benkart and S. Witherspoon, "Restricted two-parameter quantum groups", pp. 293-318 in Representations of finite dimensional algebras and related topics in Lie theory and geometry, edited by V. Dlab and C. M. Ringel, Fields Inst. Commun. 40, Amer. Math. Soc., Providence, RI, 2004. MR 2005b:17027 Zbl 1048.16020

[Benkart and Witherspoon 2004c] G. Benkart and S. Witherspoon, "Two-parameter quantum groups and Drinfel'd doubles", Algebr. and Representation Theory 7:3 (2004), 261-286. MR 2005g:17028 Zbl 1113.16041

[Benkart et al. 2003] G. Benkart, S. J. Kang, and K. H. Lee, "PBW-type bases of two-parameter quantum groups (of type $A$ )", preprint, 2003.

[Benkart et al. 2006] G. Benkart, S.-J. Kang, and K.-H. Lee, "On the centre of two-parameter quantum groups”, Proc. Roy. Soc. Edinburgh Sect. A 136:3 (2006), 445-472. MR 2007a:17019 Zbl 1106.17013

[Bergeron et al. 2006] N. Bergeron, Y. Gao, and N. Hu, "Drinfel'd doubles and Lusztig's symmetries of two-parameter quantum groups", J. Algebra 301:1 (2006), 378-405. MR 2007e:17010

[Bergeron et al. 2007] N. Bergeron, Y. Gao, and N. Hu, "Representations of two-parameter quantum orthogonal and symplectic groups", pp. 1-21 in Proceedings of the International Conference on Complex Geometry and Related Fields, edited by Z. Chen et al., AMS/IP Stud. Adv. Math. 39, Amer. Math. Soc., Providence, RI, 2007. MR 2008h:17012 Zbl 1148.17008

[De Concini and Kac 1990] C. De Concini and V. G. Kac, "Representations of quantum groups at roots of 1", pp. 471-506 in Operator algebras, unitary representations, enveloping algebras, and invariant theory (Paris, 1989), edited by A. Connes et al., Progr. Math. 92, Birkhäuser, Boston, 1990. MR 92g:17012 Zbl 0738.17008

[Drinfel'd 1987] V. G. Drinfel'd, “Quantum groups”, pp. 798-820 in Proceedings of the International Congress of Mathematicians, I (Berkeley, CA, 1986), edited by A. M. Gleason, Amer. Math. Soc., Providence, RI, 1987. MR 89f:17017

[Gelaki 1998] S. Gelaki, "Pointed Hopf algebras and Kaplansky’s 10th conjecture”, J. Algebra 209:2 (1998), 635-657. MR 99j:16024 Zbl 0922.16023

[Hu 2000] N. Hu, "Quantum divided power algebra, $q$-derivatives, and some new quantum groups", J. Algebra 232:2 (2000), 507-540. MR 2001h:16045 Zbl 0973.17019

[Hu 2004] N. Hu, "Quantum group structure associated to the quantum affine space", Algebra Colloq. 11:4 (2004), 483-492. MR 2006i:17020 Zbl 1073.17013

[Hu 2005] N. Hu, "Lyndon words, convex PBW bases and their R-matrices for the two-parameter quantum groups of $B_{2}, C_{2}, D_{4}$ types", manuscript, 2005.

[Hu and Shi 2007] N. Hu and Q. Shi, "The two-parameter quantum group of exceptional type $G_{2}$ and Lusztig's symmetries”, Pacific J. Math. 230:2 (2007), 327-345. MR 2008b:17019 Zbl 1148.17008 
[Hu and Wang 2007] N. Hu and X. Wang, "Quantizations of generalized-Witt algebra and of Jacobson-Witt algebra in the modular case", J. Algebra 312:2 (2007), 902-929. MR 2333191 Zbl 05166715

[Hu and Wang 2009] N. Hu and X. Wang, "Convex PBW-type Lyndon basis and restricted twoparameter quantum groups of type $B$ ”, preprint, 2009. arXiv $0812.3343 \mathrm{v} 4$

[Hu and Zhang 2006] N. Hu and H. Zhang, "Vertex representations of two-parameter quantum affine algebras $U_{r, s}(\widehat{\mathfrak{g}})$ : the simply-laced cases", preprint, 2006.

[Hu et al. 2008] N. Hu, M. Rosso, and H. Zhang, "Two-parameter quantum affine algebra $U_{r, s}\left(\widehat{\mathfrak{s l}}_{n}\right)$, Drinfel'd realization and quantum affine Lyndon basis", Comm. Math. Phys. 278:2 (2008), 453486. MR 2009b:17033

[Jimbo 1986] M. Jimbo, "A $q$-analogue of $U(\mathfrak{g l}(N+1))$, Hecke algebra, and the Yang-Baxter equation”, Lett. Math. Phys. 11:3 (1986), 247-252. MR 87k:17011 Zbl 0602.17005

[Kaplansky 1975] I. Kaplansky, Bialgebras, University of Chicago, 1975. MR 55 \#8087

[Kassel 1995] C. Kassel, Quantum groups, Graduate Texts in Mathematics 155, Springer, New York, 1995. MR 96e:17041 Zbl 0808.17003

[Kauffman and Radford 1993] L. H. Kauffman and D. E. Radford, "A necessary and sufficient condition for a finite-dimensional Drinfel'd double to be a ribbon Hopf algebra", J. Algebra 159:1 (1993), 98-114. MR 94d:16037 Zbl 0802.16035

[Kharchenko 1999] V. K. Kharchenko, "A quantum analogue of the Poincaré-Birkhoff-Witt theorem”, Algebra Log. 38:4 (1999), 476-507, 509. In Russian; translated in Algebra Logic 38:4 (1999), 259-276. MR 2001f:16075 Zbl 0936.16034

[Kharchenko 2002] V. K. Kharchenko, "A combinatorial approach to the quantification of Lie algebras”, Pacific J. Math. 203:1 (2002), 191-233. MR 2003b:17018 Zbl 1069.17008

[Lalonde and Ram 1995] P. Lalonde and A. Ram, "Standard Lyndon bases of Lie algebras and enveloping algebras", Trans. American Math. Soc. 347:5 (1995), 1821-1830. MR 95h:17013 Zbl 0833.17003

[Lusztig 1990a] G. Lusztig, "Finite-dimensional Hopf algebras arising from quantized universal enveloping algebra", J. Amer. Math. Soc. 3:1 (1990), 257-296. MR 91e:17009 Zbl 0695.16006

[Lusztig 1990b] G. Lusztig, "Quantum groups at roots of 1", Geom. Dedicata 35:1-3 (1990), 89113. MR 91j:17018 Zbl 0714.17013

[Lusztig 1993] G. Lusztig, Introduction to quantum groups, Progress in Mathematics 110, Birkhäuser, Boston, 1993. MR 94m:17016 Zbl 0788.17010

[Montgomery 1993] S. Montgomery, Hopf algebras and their actions on rings, CBMS Regional Conference Series in Mathematics 82, Amer. Math. Soc., Providence, RI, 1993. MR 94i:16019 Zbl 0793.16029

[Montgomery 1998] S. Montgomery, "Classifying finite-dimensional semisimple Hopf algebras", pp. 265-279 in Trends in the representation theory of finite-dimensional algebras (Seattle, WA, 1997), Contemp. Math. 229, Amer. Math. Soc., Providence, RI, 1998. MR 99k:16084 Zbl 0921. 16025

[Radford 1976] D. E. Radford, "The order of the antipode of a finite dimensional Hopf algebra is finite”, Amer. J. Math. 98:2 (1976), 333-355. MR 53 \#10852 Zbl 0332.16007

[Reshetikhin and Turaev 1990] N. Y. Reshetikhin and V. G. Turaev, "Ribbon graphs and their invariants derived from quantum groups", Comm. Math. Phys. 127:1 (1990), 1-26. MR 91c:57016 Zbl 0768.57003 
[Reshetikhin and Turaev 1991] N. Reshetikhin and V. G. Turaev, "Invariants of 3-manifolds via link polynomials and quantum groups", Invent. Math. 103:3 (1991), 547-597. MR 92b:57024 Zbl 0725.57007

[Reshetikhin et al. 1989] N. Y. Reshetikhin, L. A. Takhtadzhyan, and L. D. Faddeev, "Quantization of Lie groups and Lie algebras", Algebra i Analiz 1:1 (1989), 178-206. In Russian; translated in Leningrad Math. J. 1:1 (1990), 193-225. MR 90j:17039 Zbl 0677.17010

[Rosso 2002] M. Rosso, "Lyndon bases and the multiplicative formula for $R$-matrices", preprint, 2002 .

[Taft 1971] E. J. Taft, "The order of the antipode of finite-dimensional Hopf algebra", Proc. Nat. Acad. Sci. U.S.A. 68 (1971), 2631-2633. MR 44 \#4075 Zbl 0222.16012

Received October 16, 2008.

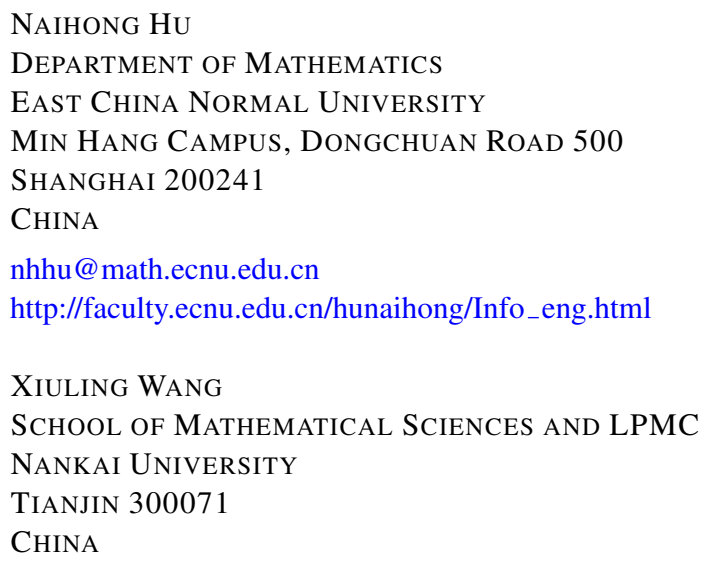

NAIHONG HU

DEPARTMENT OF MATHEMATICS

EAST CHINA NORMAL UNIVERSITY

Min Hang CAmpus, DongChuan Road 500

SHANGHAI 200241

CHINA

nhhu@math.ecnu.edu.cn

http://faculty.ecnu.edu.cn/hunaihong/Info_eng.html

\section{XiULing WANG}

SCHOOL OF MATHEMATICAL SCIENCES AND LPMC

NANKAI UNIVERSITY

TIANJIN 300071

CHINA

xiulingwang@nankai.edu.cn 